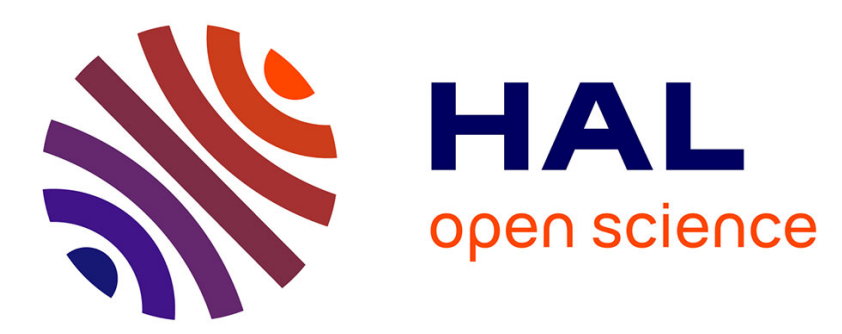

\title{
ASR Post-Correction for Spoken Dialogue Systems Based on Semantic, Syntactic, Lexical and Contextual Information
}

\author{
Ramón López-Cózar, Zoraida Callejas
}

\section{- To cite this version:}

Ramón López-Cózar, Zoraida Callejas. ASR Post-Correction for Spoken Dialogue Systems Based on Semantic, Syntactic, Lexical and Contextual Information. Speech Communication, 2008, 50 (8-9), pp.745. 10.1016/j.specom.2008.03.008 . hal-00499210

\section{HAL Id: hal-00499210 https://hal.science/hal-00499210}

Submitted on 9 Jul 2010

HAL is a multi-disciplinary open access archive for the deposit and dissemination of scientific research documents, whether they are published or not. The documents may come from teaching and research institutions in France or abroad, or from public or private research centers.
L'archive ouverte pluridisciplinaire HAL, est destinée au dépôt et à la diffusion de documents scientifiques de niveau recherche, publiés ou non, émanant des établissements d'enseignement et de recherche français ou étrangers, des laboratoires publics ou privés. 


\section{Accepted Manuscript}

ASR Post-Correction for Spoken Dialogue Systems Based on Semantic, Syn tactic, Lexical and Contextual Information

Ramón López-Cózar, Zoraida Callejas

PII:

S0167-6393(08)00036-8

DOI:

10.1016/j.specom.2008.03.008

Reference:

SPECOM 1695

To appear in:

Speech Communication

Received Date: $\quad 31$ August 2007

Revised Date: $\quad 9$ January 2008

Accepted Date: $\quad 14$ March 2008

Please cite this article as: López-Cózar, R., Callejas, Z., ASR Post-Correction for Spoken Dialogue Systems Based on Semantic, Syntactic, Lexical and Contextual Information, Speech Communication (2008), doi: 10.1016/j.specom. 2008.03.008

This is a PDF file of an unedited manuscript that has been accepted for publication. As a service to our customers we are providing this early version of the manuscript. The manuscript will undergo copyediting, typesetting, and review of the resulting proof before it is published in its final form. Please note that during the production process errors may be discovered which could affect the content, and all legal disclaimers that apply to the journal pertain. 


\title{
ASR Post-Correction for Spoken Dialogue Systems Based on Semantic, Syntactic, Lexical and Contextual Information
}

\author{
Ramón López-Cózar, Zoraida Callejas \\ Dept. of Languages and Computer Systems, Computer Science Faculty \\ 18071 Granada University, Spain, Tel.: +34 958 240579, FAX: +34 958243179 \\ E-mail: \{rlopezc, zoraida\}@ugr.es
}

\begin{abstract}
This paper proposes a technique to correct speech recognition errors in spoken dialogue systems that presents two main novel contributions. On the one hand, it considers several contexts where a speech recognition result can be corrected. A threshold learnt in the training is used to decide whether the correction must be carried out in the context associated with the current prompt type of a dialogue system, or in another context. On the other hand, the technique deals with the confidence scores of the words employed in the corrections. The correction is carried out at two levels: statistical and linguistic. At the first level the technique employs syntactic-semantic and lexical models, both contextual, to decide whether a recognition result is correct. According to this decision the recognition result may be changed. At the second level the technique employs basic linguistic knowledge to decide about the grammatical correctness of the outcome of the first level. According to this decision the outcome may be changed as well. Experimental results indicate that the technique enhances a dialogue system's word accuracy, speech understanding, implicit recovery and task completion rates by $8.5 \%$, $16.54 \%, 4 \%$ and $44.17 \%$, respectively.
\end{abstract}

Keywords: Spoken dialogue systems, speech recognition, speech understanding, natural language processing, speech-based human-computer interaction. 


\section{Introduction}

As an effort to improve automatic information services making them available 24 hours a day, 365 days a year, many companies and official institutions have recently started to employ spoken dialogue systems (McTear, 2004; López-Cózar and Araki, 2005; Kraiss, 2006; Wahlster, 2006). These systems are computer programs developed to provide specific services using speech, for example airplane travel information (Seneff and Polifroni, 2000), train travel information (Billi et al. 1997), weather forecasts (Zue et al.

2000; Nakano et al. 2001), fast food ordering (Seto et al. 1994; López-Cózar et al. 1997), call routing (Lee et al. 2000) or directory assistance (Kellner et al. 1997).

In despite of their advantages, spoken dialogue systems are rejected by many users because the interaction they allow is not very natural sometimes. This is caused by several reason, but perhaps it is mainly a consequence of the current limitations of the state-of-the-art automatic speech recognition (ASR) for real-word applications (Rabiner and Juang, 1993; Huang et al. 2001; Skantze, 2005). Hence, to make dialogue systems more widely accepted, it is very important to develop techniques to increase the robustness of the speech recogniser employed by these systems. In order to make these techniques more easily employable by the research community, we believe that the techniques will require a small effort in corpus development, they must be easily applicable to different tasks, they will require small amounts of training data, and they must be easily implemented without too much programming cost.

Many studies can be found in the literature that aim to increase the robustness of a speech recogniser, for example, Levow (1998), Swerts et al. (2000), Mangu and Padmanabhan (2001), Suhm et at. (2001), Levow (2002), Kakutani et al. (2002), Lo and Soong (2005), Ogata and Goto (2005), Shi and Zhou (2006), Denda et al. (2007) and 
Morales et al. (2007). ASR post-correction techniques aim to find incorrect words in the recognition result provided by the speech recogniser, and replace them with the correct ones, i.e. those uttered by the speaker. The techniques available so far in the literature, briefly discussed in Section 2, present several limitations. One is that many of them take into account lexical information only, and thus require large amounts of training data. Another drawback is that they not take into account the contextual information available in spoken dialogues. For example, if a spoken dialogue system prompts for a telephone number, it is likely that the user will utter specific kinds of words (digits) but not others (e.g. city names) to answer the prompt. Moreover, in a spoken dialogue the context in which words are uttered may change as the interaction proceeds. This kind of contextual information is missing in existing techniques, but according to our experiments, it can be very useful for the success of the ASR post-correction.

Another limitation of existing ASR post-correction techniques is that they consider the recogniser output just as a sequence of words, but do not take into account the confidence scores that may be attached to the words. However, many spoken dialogue systems employ these scores to decide whether to accept, reject or confirm (either implicitly or implicitly) the words in the speech recognition results (Hazen et al. 2002). The techniques available so far propose methods to replace a word $w$ in a recognition result with another word $w^{6}$ in order to make a correction, but an open question is what should be the confidence score for the word $w^{6}$ : should it be that of $w$ or a different one? The technique we present in this paper addresses the drawbacks of the existing techniques discussed above.

The remainder of the paper is organised as follows. Section 2 presents previous work related to ASR post-correction, including differences and similarities with the 
proposed technique. Section 3 focuses on this technique. It firstly discusses the required elements: word-classes, grammatical rules, syntactic-semantic models and lexical models. Secondly, it explains algorithmically how to implement the technique. Thirdly, it analyses the performance of the technique in dealing with word insertions, substitutions and deletions, and then discusses the main advantages of the technique. Section 4 presents the experiments. Firstly, it shows the interaction between the Saplen system and the user simulator, and comments on the speech database and the scenarios for the simulator. Then it comments on experiments to decide the requirements on training data. Thirdly, the section compares results obtained with the baseline system and the proposed technique, and shows the advantages of using syntactic-semantic and lexical models, both contextual. Section 5 discusses limitations of the technique. Finally, section 6 presents the conclusions and describes possibilities for future work.

\section{Previous related work}

Most previous studies on ASR post-correction are based on statistical methods that use probabilistic information about words uttered and words in the recognition results. For example, following this approach Ringger and Allen (1996) proposed a post-processor to correct speech recognition errors based on two parts. A channel model represented errors made by a speech recogniser, whereas a language model represented the likelihood of a sequence of words uttered by the speaker. They trained both models with transcriptions of dialogues obtained with the TRAINS-95 dialogue system. Their experimental results showed that the post-processor output contained fewer errors than that of the speech recogniser. Also following this approach, Zhou and Meng (2004) proposed a two-level schema for detecting speech recognition errors. The first level applied an utterance classifier to decide whether the speech recognition result was 
erroneous. If it was determined to be incorrect, it was passed to the second level where a word classifier decided which words were misrecognitions.

Other methods employ co-occurrence information extracted from the words and their neighbouring words. For example, Zhou et al. (2006) proposed a method for error detection based on three steps. The first step was to detect whether the input utterance was correct. The second step was to detect incorrect words, and the third step was to detect erroneous characters. The error correction first created candidate lists of errors, and then re-ranked the candidates with a model that combines mutual information and a word trigram.

The methods discussed so far present several drawbacks. One is that they require large amounts of training data. Another is that their success depends on the size and quality of the speech recognition results or on the database of collected error strings, since they are directly dependent on the lexical entries. To address these drawbacks, researchers have employed additional knowledge sources. For example, Jeong et al. (1996) combined lexical information with semantic knowledge and carried out error correction at two levels: semantic and lexical. The input utterance was firstly transformed to obtain a lexico-semantic pattern. A database of pre-collected semantic patterns was used at the semantic level to find similar patterns to the obtained pattern. The error correction was made by replacing erroneous syntactic or semantic items in the obtained pattern, taking into account the pre-collected similar patterns. At the lexical correction level, the obtained and the recovered patterns were aligned, some candidate words in a domain dictionary or ontology dictionary were selected as the most similar to the original input words, and these words were used for correction. 
Employing a different approach, Jeong et al. (2004) combined lexical information with higher level knowledge sources via a maximum entropy language model (MELM). Error correction was arranged on two levels, using a different language model at each level. At the first level, a word n-gram was employed to capture local dependencies and to speed up the processing. The MELM was used at the second level to capture long-distance dependencies and higher linguistic phenomena, and to re-score the N-best hypotheses produced by the first level. Their experiments showed that this approach had superior performance than previous lexical-oriented approaches. The problem was that the training of the MELM required a lot of time and was sometimes infeasible.

\subsection{Differences and similarities between the proposed technique and previous studies}

The technique we propose is inspired by previous studies based on semantic information (Jeong et al. 1996), pattern matching (Kaki et al. 1998) and statistical information (Zhou and Meng, 2004). One similarity between our technique and that of Jeong et al. (1996) is in the use of two correction levels. In both techniques the speech recognition result is transformed into one pattern. At the first level, this pattern is compared with a corpus of patterns learnt in the training, and as a result of the comparison the input pattern may be changed. The outcome of this level is passed on to the second level where both techniques can replace some words with other words.

One difference between both techniques is in the method employed to select the pattern to be used for making corrections at the first level. According to the technique of Jeong et al. (1996), this pattern is the one with minimum distance to the input pattern. One problem of this method is that the selected pattern may not be optimal. To 
overcome this problem, our method employs several corpora of previously learnt patterns, and a similarity threshold $t \in[0.0-1.0]$ to decide whether one pattern is good enough for error correction. If it is, our method works similarly to that of Jeong et al. (1996); otherwise it searches for a better pattern in the whole set of patterns available. If the appropriate pattern is found in the whole set, the correction proceeds as in the method of Jeong et al. (1996); otherwise our technique does not make any correction at the first level. Another difference is that Jeong et al. (1996) carry out the lexical correction at the second level, whereas our method carries this correction both at the first and second levels. In the former level it considers statistical information, while in the latter it takes into account linguistic knowledge.

Our technique also has similarities with the proposal of Zhou and Meng (2004), as both employ a two-level schema for detecting recognition errors. The first level decides whether the speech recognition result is erroneous. If it is, the technique of Zhou and Meng (2004) passes on the result to the second level, where a word classifier decides which words are incorrect. One difference between the two techniques is that ours always passes on the result of the first level to the second level, regardless of the decision made by the first level.

Another difference between our technique and previous studies is that, as discussed in Section 1, existing studies focus just on the words in the speech recognition result, without considering the confidence scores that the words may have attached. Our technique not only deals with the word strings, but also with the confidence scores. As far as we know, this is an issue not addressed in previous studies. 


\section{The proposed technique}

We propose a new ASR post-correction technique for spoken dialogue systems that can be useful when the speech recognition rate is very low. This is typically the case when such a system employs just one prompt-independent language model (e.g. word bigram) to recognise, in theory, any kind of sentence uttered by the user within the application domain, regardless of the current prompt generated by the system. Hence, our goal is to move forward from the typical solution employed by commercial systems, i.e. promptdependent language models mostly, to a more ambitious and less restricted interaction by means of just one prompt-independent language model, which enables the user to say anything within the application domain at anytime. We assume that the technique must be applicable to any dialogue system, regardless of the speech recogniser employed. Hence, we consider the recogniser as a black box that for each input utterance produces a recognition result. This result is a sequence of words with confidence scores ${ }^{1}$ attached, e.g. "I (0.7590) would (0.6982) like (0.9268) to (0.4285) have (0.6929) six (0.3974) green (0.7059) salads $(0.8182)$ ". If the recogniser does not produce these scores but only the word sequence, our technique is applicable as well by simply discarding all the decisions about the scores.

The technique employs semantic, syntactic, lexical and contextual information. The semantic and syntactic information is implemented by means of sets of patterns constructed from the analysis of a dialogue corpus. Each set is mapped to a prompt type of a spoken dialogue system designed for a given application. The prompt type represents the contextual information of the technique, as it determines the kinds of

\footnotetext{
${ }^{1} \mathrm{We}$ assume in this paper that the confidence scores are real numbers in the range $[0.0-1.0]$.
} 
sentence likely to be uttered by the user at a particular dialogue state. The lexical information is implemented by means of word confusion statistics and linguistic knowledge. The statistics are computed from the analysis of the dialogue corpus, by aligning utterance transcriptions and speech recognition results. The linguistic knowledge must be provided by the system designers and is employed to ensure grammatical restrictions (e.g. number correspondences) between specific words, in case these restrictions affect the semantics of the sentences. The reason for using this knowledge is to compensate for problems arising from sparse training data.

\subsection{Elements to be created for applying the technique}

If we want to apply the technique to a spoken dialogue system, we must create the following elements: word-classes, grammatical rules, syntactic-semantic models and lexical models. The creation of these elements requires a corpus of system-user dialogues $^{2}$ that contains user utterances (voice samples files), transcriptions of the utterances (text sentences), and speech recognition results (text sentences) obtained by the speech recogniser of the dialogue system as it analyses the utterances.

We must assign a type (T) to each prompt the dialogue system can generate, taking into account the kind of data expected when the user answers the prompt. Note that a system may generate a prompt in different ways to avoid redundancy and increase the naturalness in the dialogue. For example, in the fast food domain a system can generate prompts such as "Would you like to have anything to eat?", "Anything to eat?" or "Are you ordering anything to eat?", but regardless of the wording, what is expected from the

\footnotetext{
${ }^{2}$ This dialogue corpus can also be obtained by employing a user simulator that interacts with the dialogue system, as we have done in the experiments.
} 
user is either a confirmation or a food order. Table 1 shows some assignments of types to prompts generated by the Saplen system used in the experiments.

Table 1. Assignment of types to prompts generated by the Saplen system.

\begin{tabular}{ll}
\hline \multicolumn{1}{c}{ Prompt } & \multicolumn{1}{c}{ Type (T) } \\
\hline Please say your telephone number. & TELEPHONE_NUMBER \\
I'm sorry, I didn't understand. Please say your telephone number again. & TELEPHONE_NUMBER \\
What would you like to have? & PRODUCT_ORDER \\
How many ham sandwiches did you say? & FOOD_NUMBER \\
Which size would you like for the beer? & DRINK_SIZE \\
Did you say two? & NUMBER_CONFIRMATION \\
Please say the taste for the milkshake. & DRINK_TASTE \\
Do you want to remove the green salad? & REMOVE_CONFIRMATION \\
Did you say large? & SIZE_CONFIRMATION \\
\hline
\end{tabular}

\subsubsection{Word-classes}

The word-classes $\mathrm{K}_{\mathrm{i}}$ are created using the set of utterance transcriptions in the dialogue corpus. Each word-class contains words of a given type that are really useful for the semantic analyser of the dialogue system to get the meaning of the sentences uttered by the user. These words are usually called keywords. The word-classes to be created can be decided by observing the transcriptions and using the system designers' knowledge about the performance of the semantic analyser. We call $\Omega$ the set of word-classes: $\Omega=$ $\left\{\mathrm{K}_{1}, \mathrm{~K}_{2}, \mathrm{~K}_{3}, \ldots, \mathrm{K}_{\mathrm{r}}\right\}$. Table 2 shows some possible word-classes in the fast food domain. Table 2. Examples of word-classes in the fast food domain.

\begin{tabular}{ll}
\hline Word-class & \multicolumn{1}{c}{ Word examples } \\
\hline CONFIRMATION & yes, no, OK, correct, incorrect, alright, ... \\
DESIRE & want, need, gimme, ... \\
DRINK & water, beer, coke, wine, fanta, ... \\
FOOD & sandwich, cake, ice-cream, burger, ... \\
INGREDIENT & cheese, ham, bacon, ... \\
NUMBER & one, two, three, four, five, six, ... \\
SIZE & small, large, big, ... \\
TASTE & orange, lemon, apple, ... \\
\hline
\end{tabular}

Usually a keyword belongs to just one word-class, e.g. 'want' belongs to the DESIRE class only. There may be keywords that belong to several word-classes, e.g. 'orange' could belong to the FOOD and TASTE classes. The technique requires the creation of 
word-classes for keywords only. Hence, word-classes are not required for meaningless words (e.g. articles or prepositions) if these words are not relevant for the semantic analyser of the dialogue system.

The word-classes are task-dependent. For example, in the ATIS (Air Travel Information Service) domain we could create the word-classes shown in Table 3.

Table 3. Examples of word-classes in the ATIS domain.

\begin{tabular}{ll}
\hline Word-class & \multicolumn{1}{c}{ Word examples } \\
\hline DESIRE & want, need, ... \\
CITY & Boston, Chicago, Rome, Madrid, Tokyo, ... \\
WEEK_DAY & Monday, Tuesday, Wednesday, ... \\
MONTH_DAY & first, second, third, fourth, ... \\
MONTH_NAME & January, February, March, April, ... \\
NUMBER & one, two, three, four, five, six, ... \\
ACTION & book, cancel, confirm, question, ... \\
DEPARTURE & from, departure, departs, ... \\
ARRIVAL & arrive, arrives, arrival, ... \\
\hline
\end{tabular}

The effort of determining the required word-classes for a given application may be relatively small using the designers' knowledge of the system, but filling the classes with all the required words can be costly if this is done manually, i.e. inserting the keywords one by one. However, if the semantic analyser of the dialogue system already uses word-classes to extract the meaning of the sentences uttered by the users, the technique can use these classes and there is no need to create them manually. This is the case for the Saplen system used in the experiments, where the semantic analyser uses word-classes stored in text files (one file per word-class). If the dialogue system does not use word-classes but uses finite-state grammars, which is the case for many commercial systems based on VoiceXML, it is possible to save time in creating the word-classes by re-using the vocabulary in the grammars, which is usually arranged in word categories. For example, Fig. 1 shows a JSGF grammar employed in a VoiceXML system that provides travel information. City names or week days can be easily copied and pasted to create the word-classes CITY and WEEK_DAY. 
\#JSGF V1.0;

grammar from_to;

public $<$ from_to $>=[$ greeting $]<$ desire $><$ travel $><$ city $>\{$ this.cityDestination=\$city $\}<$ from_mark $>$ $<$ city $>\{$ this.cityDeparture $=\$$ city $\}<$ moment $>$ \{this.weekDay=\$moment $\}$;

$<$ greeting $>=$ hello $\mid$ hi $\mid$ good morning $\mid$ good evening ;

$<$ desire $>=$ ( I | we ) | ( want I desire I need I must ) ;

$<$ travel $>=$ go to $\mid$ travel to $\mid$ get to ;

$<$ city $>=$ New York | Boston | Chicago | London | Madrid | Paris | Rome ;

$<$ from_mark $>$ = from I leaving from I departing from ;

$<$ moment $>=($ this I next ) ( Monday | Tuesday | Wednesday | Thursday I Friday I Saturday | Sunday );

Fig. 1. A sample JSGF grammar.

\subsubsection{Grammatical rules}

The grammatical rules are used to correct grammatical errors that affect the semantics of sentences. We call $\mathrm{R}$ the set of basic grammatical rules $r_{\mathrm{i}}: \mathrm{R}=\left\{r_{1}, r_{2}, \ldots, r_{\mathrm{n}}\right\}$. The rules are of the form: $r_{\mathrm{i}}: s s p_{\mathrm{i}} \rightarrow$ restriction $_{\mathrm{i}}$, where $s s p_{\mathrm{i}}$ is a syntactic-semantic pattern and restriction $n_{\mathrm{i}}$ is a condition that must be satisfied by the words represented by the word-classes in $s s p_{\mathrm{i}}$. For example, one rule used in our experiments in the fast food domain is the following:

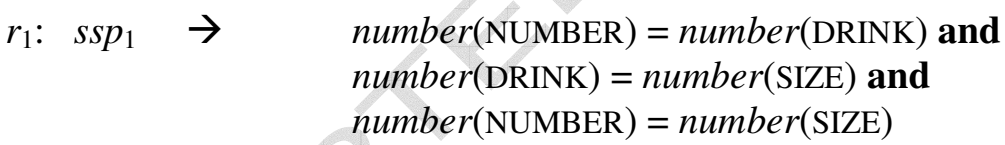

where number is a function that returns either 'singular' or 'plural' for each word in the word-class, and $s s p_{1}=$ NUMBER DRINK SIZE.

One drawback of this method is that the rules are task-dependent. Hence, if the application domain is changed, the rules must be adapted accordingly to consider the necessary grammatical restrictions among the word-classes in the new domain. One alternative is to use statistical information, which has the advantage of automatic training without manual effort to create grammatical rules. To study the differences between both methods, we followed the study by Zhou et al. (2006) and used one n- 
gram language model, i.e. the word bigram employed by the speech recogniser of the Saplen system. We found that in some cases the statistical method worked well in correcting errors in speech recognition results, e.g. in "uno cervezas grandes" (one large beers), where 'uno' (one) was a misrecognition of 'dos' (two). The probability of this speech recognition can be expressed as follows:

$$
P(\text { uno cervezas grandes })=P(\text { uno }) \times P(\text { cervezas } \mid \text { uno }) \times P(\text { grandes } \mid \text { cervezas })
$$

Using the bigram we found that the probabilities $P$ (uno) and $P$ (grandes|cervezas) were large as the word sequences 'uno' and 'cervezas grandes' were observed quite frequently in the training corpus. On the contrary, the probability $P$ (cervezas|uno) was very small as the word sequence 'uno cervezas' was not observed. Thus we assumed that 'uno' was an incorrect word. We searched for an appropriate word in the trained lexical model (as will be explained in Section 3.1.4) and found the perfect candidate: 'dos' (two). Hence, we replaced 'uno' with 'dos' and had the misrecognition correctly corrected: "dos cervezas grandes" (two large beers).

The problem with this method is that because of sparse training data, there were cases where the method transformed correct recognition results into incorrect results, which is a known problem of for purely statistical methods as observed in previous studies, e.g. Kaki et al. (1998) and Zhou et al. (2006). For example, in our application domain this happened with the recognition result "diez cervezas grandes" (ten large beers), which was not erroneous. For this result we found in the word bigram that the probabilities $P($ diez) and $P$ (grandes|cervezas) were large, as the word sequences 'diez' and 'cervezas grandes' were observed, whereas the probability $P$ (cervezas|diez) was very small as the word sequence 'diez cervezas' was not observed. Then we assumed, as in the above example, that 'diez' was an incorrect word. Hence, we searched for an appropriate 
substitution in the lexical model and found the candidate: 'tres'. Therefore, we replaced 'diez' with 'tres' and had the recognition result incorrectly corrected: "tres cervezas grandes" (three large beers).

Employing a large training corpus, we could have implemented this statistical method for making the correction at the lexical level, instead of the one based on grammatical rules. We decided to use the latter as it works better in cases of sparse data, given that the rules apply to all the words in the word-classes, regardless of whether the words have been observed in the training. Using the rule-based approach, the word 'diez' (ten) in the example above would not have been considered an incorrect word as long as it is in the word-class NUMBER.

\subsubsection{Syntactic-semantic models}

The syntactic-semantic models are representations of the conceptual structure of the sentences in the application domain. To create these models we consider each prompt type $\mathrm{T}$ and take into account the transcriptions of all the sentences uttered by the users to answer system prompts of type $\mathrm{T}$. For example, for the prompt type $\mathrm{T}=$ TELEPHONE_NUMBER we could find transcriptions such as: "My telephone number is nine five eight one two three four five six", "nine five eight one two three four five six" or "nine five eight twelve thirty-four fifty-six".

Each transcription must be transformed into what we call a syntactic-semantic pattern (ssp), which represents the sequence of word-class names in the transcription. We say that this kind of pattern is syntactic as it provides information about the order of the word-classes in the sequence. For example, the two $s s p$ 's: $s s p_{1}=$ DESIRE NUMBER INGREDIENT and $s s p_{2}=$ NUMBER DESIRE INGREDIENT are syntactically different as they differ in the order of the word-classes. We say that this kind of pattern is also 
semantic as it provides information about the concepts (represented as word-classes) employed to convey some semantic content. For example, the two $s s p$ 's: $s s p_{1}=$ DESIRE NUMBER FOOD and $s s p_{2}=$ DESIRE NUMBER DRINK are semantically different as they differ in the concepts involved ( $s p_{1}$ is a conceptual representation of a food order, whereas $s s p_{2}$ is a conceptual representation of a drink order).

To create a ssp from a transcription, each keyword in the transcription must be replaced with the name of the word-class the keyword belongs to. For example, taking into account the word-classes shown in Table 2 (Section 3.1.1), the ssp for the transcription: "I want one ham sandwich and one green salad please", would be as follows: $s s p=$ DESIRE NUMBER INGREDIENT NUMBER SIZE DRINK

If a keyword belongs to several word-classes, we include the names of the wordclasses within brackets and separate them with the optional marker 'l'. For example, if the keyword 'orange' belongs to the classes FOOD and TASTE, the ssp for the transcription "I want one orange Fanta" would be:

$s s p=$ DESIRE NUMBER ( FOOD | TASTE ) DRINK

Transforming all the utterance transcriptions associated with a prompt type $\mathrm{T}$ into ssp's, we obtain a set of ssp's associated with T. The next step is to analyse this set to remove repeated ssp's, associating with each different $s s p$ its relative frequency within the set. We call the result of this process a syntactic-semantic model associated with the prompt type $\mathrm{T}\left(\mathrm{SSM}_{\mathrm{T}}\right)$. This model is as follows:

$$
\mathrm{SSM}_{\mathrm{T}}=\left\{s s p_{1} \mathrm{f}_{1}, s s p_{2} \mathrm{f}_{2}, s s p_{3} \mathrm{f}_{3}, \ldots, s s p_{\mathrm{m}} \mathrm{f}_{\mathrm{m}}\right\}
$$

where the $s s p_{\mathrm{i}}{ }^{\text {'s }}$ are syntactic-semantic patterns, and the $\mathrm{f}_{\mathrm{i}}$ 's are their relative frequencies within $\mathrm{SSM}_{\mathrm{T}}$. For example, the $\mathrm{SSM}_{\mathrm{T}}$ associated with the prompt type $\mathrm{T}=$ TELEPHONE_NUMBER in our experiment is as follows: 
SSM

$\{$ NUMBER NUMBER NUMBER NUMBER NUMBER NUMBER 0.1430 ,

NUMBER NUMBER NUMBER NUMBER NUMBER NUMBER NUMBER NUMBER NUMBER 0.1430,

NUMBER SIZE TASTE DRINK 0.0173,

MISTAKE 0.0098,

NUMBER INGREDIENT FOOD 0.0098,

...

If a dialogue system generates $u$ different prompt types $\mathrm{T}$, we must create $u$ different syntactic-semantic models $\mathrm{SSM}_{\mathrm{T}}$, one per prompt type. We call $\alpha$ set of all the $\mathrm{SSM}_{\mathrm{T}}$ 's for a given dialogue system:

$$
\alpha=\left\{\mathrm{SSM}_{\mathrm{Ti}}\right\}, \mathrm{i}=1 \ldots u
$$

Note that a $\mathrm{SSM}_{\mathrm{T}}$ is created from sentences uttered by users to answer a prompt type $\mathrm{T}$. Hence, it is expected that the model contains syntactic-semantic patterns obtained from sentences actually related to the prompt type $\mathrm{T}$, for example, patterns obtained from telephone numbers if the system prompted for a telephone number. However, a $\mathrm{SSM}_{\mathrm{T}}$ can also contain ssp's not directly related to the prompt type, which happens if the users uttered other types of sentence. For example, the SSM TELEPHONE_NUMBER shown above contains a $s s p$ obtained from food orders (NUMBER INGREDIENT FOOD 0.0098), another obtained from drink orders (NUMBER SIZE TASTE DRINK 0.0173) and another obtained from user corrections to system misunderstandings (MISTAKE 0.0098).

\subsubsection{Lexical models}

The lexical models contain information about the performance of the dialogue system's speech recogniser at each specific dialogue state. This state is represented by the prompt type $\mathrm{T}$ of the system. We must create a lexical model $\left(\mathrm{LM}_{\mathrm{T}}\right)$ for each prompt type. The general form of this model is as follows:

$$
\mathrm{LM}_{\mathrm{T}}=\left\{\begin{array}{lll}
w_{i} & w_{j} & p_{i j}
\end{array}\right\} \quad i, j=1 \ldots s
$$

where $w_{i}$ is a word uttered by the speaker, $w_{j}$ is the recognition result, and $p_{i j}$ is the posterior probability of obtaining $w_{j}$ when the speaker uttered $w_{i}$. To create a $\mathrm{LM}_{\mathrm{T}}$ we 
employ the set of utterance transcriptions and the set of recognition results associated with the prompt type T. Both sets are available from the analysis of the dialogue corpus. We align each transcription $c$ with its corresponding recognition result $h$ following the study of Fisher and Fiscus (1993), and add $\left(w_{i}, w_{j}\right)$ pairs to $\mathrm{LM}_{\mathrm{T}}, w_{i} \in c, w_{j} \in h$. When all the transcriptions have been aligned, $\mathrm{LM}_{\mathrm{T}}$ is analysed to remove repeated entries and to compute the probabilities $p_{i j}$. For example, let us suppose that the dialogue system prompted for the user's telephone number, i.e. T = TELEPHONE_NUMBER, and that to answer this prompt the user uttered the sentence: "nine five eight one two three four five six". Let us assume that the recognition result from this utterance is: "mine (0.3841) five (0.7867) eight $(0.9345)$ one $(0.7810)$ two $(0.6721)$ three $(0.8945)$ four $(0.7832)$ five $(0.7903)$ six (0.3981)". Then, following the procedure discussed above, the word pairs: ('nine' , 'mine'), ('five', 'five'), ('eight', 'eight'), ('one' , 'one'), ('two', 'two'), ('three', 'three'), ('four' , 'four'), ('five' , 'five') and ('six', 'six') are added to $\mathrm{LM}_{\mathrm{T}}$. Hence, $\mathrm{LM}_{\mathrm{T}}$ contains statistical information about the recognition of each word uttered by the user in response to the prompt type. After the removal of repeated entries and the computation of the word recognition probabilities, $\mathrm{LM}_{\text {TELEPHONE_NUMBER }}$ may be as follows:

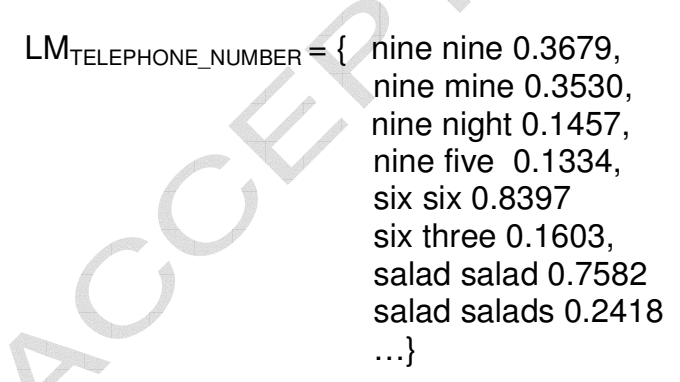

If a dialogue system generates $u$ different prompt types $\mathrm{T}$, we must create $u$ different lexical models $\mathrm{LM}_{\mathrm{T}}$, one per prompt type. We call $\beta$ the set of all the $\mathrm{LM}_{\mathrm{T}}$ 's for a given dialogue system:

$$
\beta=\left\{\mathrm{LM}_{\mathrm{Ti}}\right\}, \mathrm{i}=1 \ldots u
$$


As discussed for the syntactic-semantic models in the previous section, a $\mathrm{LM}_{\mathrm{T}}$ is created from sentences uttered by users in the answering of system prompts of type T. Hence, it is expected that the model contains word recognition probabilities obtained from utterances actually related to the prompt type $\mathrm{T}$, for example, recognition probabilities of digits if the system prompted for a telephone number. However, $\mathrm{LM}_{\mathrm{T}}$ can also contain word recognition probabilities obtained from utterances not directly related to the prompt type, which happens if the users uttered other types of sentence. For example, the LM $\mathrm{M}_{\text {TELEPHONE_NUMBER }}$ shown above contains recognition probabilities for the word 'salad', which in principle is not expected when the system prompted for the user's telephone number.

\subsection{Algorithms to implement the technique}

The ASR post-correction technique proposed in this paper is carried out firstly at the statistical level, and then at the linguistic level. The correction at the former level deals with the confidence scores if these scores are observed in the input utterance, otherwise the decision about these scores is skipped.

\subsubsection{Correction at the statistical level}

The goal of the correction at the statistical level is to take the result of the speech recogniser and employ statistical information to find words that belong to incorrect concepts, replace these concepts with correct concepts, and select the appropriate words for the correct concepts. If the algorithm finds that a word $w$ belongs to an incorrect concept $\mathrm{J}$ and decides to replace it with another word $w$ ' that belongs to another concept $\mathrm{K}$, it must decide the confidence score for the correction word $w^{\prime}: C\left(w^{\prime}\right)$. To do this we propose a simple method that takes into account the number of words $u_{\mathrm{j}} \in \mathrm{K}$ that are in the lexical model employed (either $\mathrm{LM}_{\mathrm{T}}$ or $\beta$ ), i.e. words with which the word $w$ is 
confused. These words form a set $\mathrm{U}=\left\{u_{1}, u_{2}, u_{3}, \ldots, u_{\mathrm{p}}\right\}$. The method to determine $C\left(w^{\prime}\right)$ is as follows: if there is just one word $u_{\mathrm{j}}$ in $\mathrm{U}$, then $w^{\prime}=u_{\mathrm{j}}$ and $C\left(w^{\prime}\right)=1.0$. If there are several words $u_{\mathrm{j}}{ }^{\prime} \mathrm{s}$ in $\mathrm{U}$, then $w^{\prime}$ is the word with the highest confusion probability: $p$, and $C\left(w^{\prime}\right)=p$. This method assigns to $w^{\prime}$ the highest confidence score (1.0) if there is just one candidate word to make the word replacement, and assigns a smaller value if there are several candidates. We do not claim that his method is optimal, and in future work we will study other methods.

To implement the correction, the algorithm receives the speech recognition result and builds what we call an input enriched syntactic-semantic pattern (essp INPUT), which is a sequence of information containers $C_{i}$ as shown in Fig. 2:

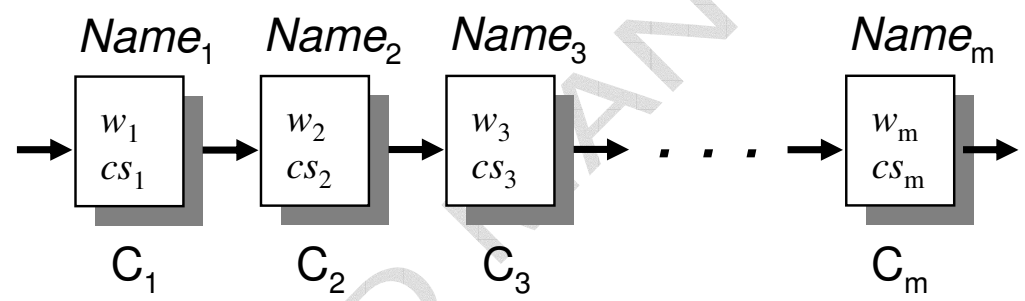

Fig. 2. General format of an input enriched syntactic-semantic pattern (essp $\left.p_{\text {INPUT }}\right)$.

Each container has an optional name which is the name of the word-class that contains the word $w_{\mathrm{i}}$ in the container. If this word is not in any word-class, the container has no name. If a container has a name, we say it is a conceptual container. The $w_{\mathrm{i}}$ 's are the words in the speech recognition result, whereas the $c s_{\mathrm{i}}$ 's are the confidence scores of the words. For example, using the word-classes shown in Table 2 (Section 3.1.1), the $\operatorname{ess}_{\text {INPUT }}$ obtained from the recognition result: "I (0.5735) want $(0.7387)$ one $(0.6307)$ ham (0.3982) sandwich (0.6307) and (0.4530) one (0.6854) small (0.6590) beer (0.7861)" would be as shown in Fig. 3. Note that all the containers are conceptual, except the first and the sixth. 


\section{ACCEPTED MANUSCRIPT}

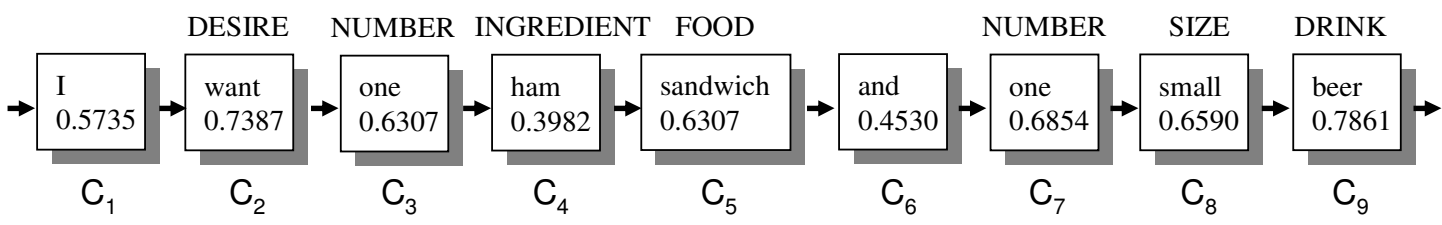

Fig. 3. Example of input enriched syntactic-semantic pattern (essp $\left.p_{\text {INPUT }}\right)$.

The algorithm now carries out two steps: pattern matching and pattern alignment, which are discussed below.

\section{Step 1. Pattern matching}

The goal of this task is to create an enriched syntactic-semantic pattern that we call $\operatorname{ess}_{\mathrm{BEST}}$. To do this we firstly define $\operatorname{ssp}_{\text {INPUT }}$ as the sequence of word-class names $\mathrm{N}_{\mathrm{i}}$ in $e s s p_{\text {INPUT }}$ For example, $s s p_{\text {INPUT }}$ for the $e s s p_{\text {INPUT }}$ shown in Fig. 3 would be:

$s s p_{\text {INPUT }}=$ DESIRE NUMBER INGREDIENT NUMBER SIZE DRINK

We decide whether $s s p_{\text {INPUT }}$ matches any of the $s s p$ 's in the syntactic-semantic model associated with the prompt type $\mathrm{T}\left(\mathrm{SSM}_{\mathrm{T}}\right)$. If it does, we set $e_{\text {ssp }} p_{\mathrm{BEST}}=e s s p_{\mathrm{INPUT}}$ and proceed to the correction at the linguistic level. In this case Step 2 is not necessary as no changes have been made in the sequence of concepts (i.e. word-class names) in $s s p_{\text {INPUT. If }} s s p_{\text {INPUT }}$ does not match any pattern in $\mathrm{SSM}_{\mathrm{T}}$, we search for similar patterns to $s s p_{\text {INPUT }}$ in $\mathrm{SSM}_{\mathrm{T}}$. To do this we compare $s s p_{\text {INPUT }}$ with all the $s s p$ 's in $\mathrm{SSM}_{\mathrm{T}}$ employing the minimum edit-distance dynamic $\operatorname{search}^{3}$ (Crestani, 2000), and select

\footnotetext{
3 The minimum edit distance between two syntactic-semantic patterns is defined as the number of deletions, insertions and substitutions of word-class names required for transforming one pattern into the other.
} 
those with similarity value greater than a threshold ${ }^{4} t \in[0.0-1.0]$. We compute the similarity of one pattern $s s p_{1}$ with respect to the other pattern $s s p_{2}$ as follows:

$$
\operatorname{similarity}\left(s s p_{1}, s s p_{2}\right)=\left(n-\mathrm{m}_{\mathrm{ed}}\right) / n
$$

where $n$ is the number of word-class names in $s s p_{1}$, and $\mathrm{m}_{\mathrm{ed}}$ is the minimum edit distance between both patterns. Note that if $s s p_{1}$ and $s s p_{2}$ are exactly the same, their similarity is 1.0 given that $\mathrm{m}_{\mathrm{ed}}=0$. If they are completely different, their similarity is 0.0 because $\mathrm{m}_{\mathrm{ed}}=n$. We call $s s p_{\mathrm{SIMILAR}}$ each $s s p$ in $\mathrm{SSM}_{\mathrm{T}}$ such that similarity ( $s p_{\text {INPUT }}$, $s s p)>t$, and consider three cases:

Case 1. There is just one $s s p_{\text {SIMILAR }}$ in $\mathbf{S S M}_{\mathbf{T}}$. In this case the correction is made by setting $s s p_{\mathrm{BEST}}=s s p_{\mathrm{SIMILAR}}$ and proceeding to Step 2 , which is now necessary as the sequence of concepts (i.e. word-class names) in $s s p_{\mathrm{BEST}}$ is different from that in $s s p_{\text {INPUT }}$

Case 2. There are no $s s p_{\text {SIMILAR's in }} \mathbf{S S M}_{\mathrm{T}}$. This means that the $s s p$ obtained from the recognition result is very different from all the ssp's in $\mathrm{SSM}_{\mathrm{T}}$. Then, we follow a fallback strategy and try to find $s s p_{\text {SIMILAR }}$ 's in the $\alpha$ set (see Section 3.1.3). If no $s s p_{\text {SIMILAR }}$ 's are found in $\alpha$, this means that the obtained $s s p$ is very different from all the ssp's observed in the training, regardless of the system prompt type T. In this case we do not make any correction and proceed to the correction at the linguistic level. If just one $s s p_{\text {SIMILAR }}$ is found in $\alpha$, the correction is made as in Case 1 , i.e. setting $s s p_{\text {BEST }}=$ $s s p_{\text {SIMILAR }}$ and proceeding to Step 2 . If several $s s p_{\text {SIMILAR }}$ 's are found in $\alpha$, we proceed as in Case 3.

\footnotetext{
${ }^{4}$ The optimal value of the similarity threshold must be determined experimentally, employing the technique over a small set of the test database, and selecting as optimal the value that provides the best performance.
} 
Case 3. There are several $\operatorname{ssp}_{\text {SIMILAR }}{ }^{6} \mathbf{s}$ in $\mathbf{S S M}_{\mathbf{T}}$ or $\boldsymbol{\alpha}$. The question now is to decide the best $s s p_{\text {SIMILAR }}$ to make the correction. To do this we start by selecting all the $s s p_{\text {SIMILAR }}$ 's with the greatest similarity value. If there is just one, we set $s s p_{\mathrm{BEST}}=$ $s s p_{\text {SIMILAR }}$ and proceed to Step 2. If there are several, we select among them those with the highest relative frequency $f_{i}$ in $\mathrm{SSM}_{\mathrm{T}}$ or $\alpha$. If there is just one, we set $s s p_{\mathrm{BEST}}=$ $s s p_{\text {SIMILAR }}$ and proceed to Step 2 . If there are several, we do not make any correction at the statistical level and proceed to the correction at the linguistic level.

\section{Step 2. Pattern alignment}

Up to this point we haven taken $s s p_{\text {INPUT }}$ and have created $s s p_{\text {BEST. The former is of the }}$ form: $s s p_{\text {INPUT }}=\mathrm{N}_{1} \mathrm{~N}_{2} \ldots \mathrm{N}_{\mathrm{m}}$, for example: $s s p_{\text {INPUT }}=$ DESIRE INGREDIENT SIZE DRINK, whereas the latter is of the form: $s s p_{\mathrm{BEST}}=\mathrm{M}_{1} \mathrm{M}_{2} \quad \ldots \quad \mathrm{M}_{\mathrm{r}}$, for example: $s s p_{\mathrm{BEST}}=$ DESIRE NUMBER SIZE DRINK. The goal of Step 2 is to create an enriched syntactic-semantic

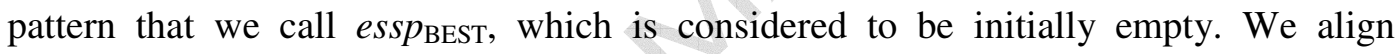
$s s p_{\text {INPUT }}$ and $s s p_{\text {BEST }}$ and focussing on each container $C_{\mathrm{i}}$ in $e s s p_{\text {INPUT }}$, we study two cases:

Case A. $\mathbf{C}_{\mathbf{i}}$ is not conceptual. In this case the word $w_{\mathrm{i}}$ in $\mathrm{C}_{\mathrm{i}}$ does not affect the semantics of the sentence, for example ' $\mathrm{I}$ ' in container $\mathrm{C}_{1}$ or 'and' in container $\mathrm{C}_{6}$ of Fig. 3. Hence, we do not try to correct $w_{\mathrm{i}}$. We simply set $\mathrm{D}_{\mathrm{i}}=\mathrm{C}_{\mathrm{i}}$ and add $\mathrm{D}_{\mathrm{i}}$ to essp $p_{\mathrm{BEST}}$ as observed in Fig. 4:

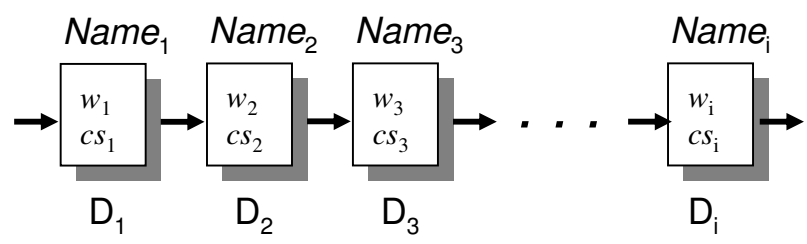

Fig. 4. $e s s p_{\text {BEST }}$ with a new container $\mathrm{D}_{\mathrm{i}}$ added. 
Case B. $\mathbf{C}_{\mathbf{i}}$ is conceptual. In this case the word $w_{\mathrm{i}}$ in $\mathrm{C}_{\mathrm{i}}$ affects the semantics of the sentence, for example 'one' in container $\mathrm{C}_{3}$ of Fig. 3 . Hence, we study whether this word must be changed considering the ssp's observed in the training. Let us say that this container is $\mathrm{N}_{\mathrm{a}}, \mathrm{a} \in 1 \ldots \mathrm{m}$, e.g. INGREDIENT in the $\operatorname{ssp}_{\text {INPUT }}$ shown above. We try to find the concept aligned with $\mathrm{N}_{\mathrm{a}}$ in $s s p_{\text {BEST. }}$. Let us say that it is $\mathrm{M}_{\mathrm{b}}, \mathrm{b} \in 1 \ldots \mathrm{r}$, e.g. NUMBER in the sample $s s p_{\mathrm{BEST}}$ shown above. We must consider three cases:

Case B.1. $\mathbf{N}_{\mathbf{a}} \neq \mathbf{M}_{\mathbf{b}}$. This is the case when a concept obtained from the speech recognition result $\left(\mathrm{N}_{\mathrm{a}}\right)$ is considered to be incorrect and then must be replaced with another concept $\left(\mathrm{M}_{\mathrm{b}}\right)$. We must decide the word $w_{\mathrm{i}}^{\prime} \in \mathrm{M}_{\mathrm{b}}$ and the confidence score $s c_{\mathrm{i}}$ ' for the new container $\mathrm{D}_{\mathrm{i}}$ to be added to $e s s p_{\text {BEST. To find }}$ this word we use the lexical model associated with the prompt type $\mathrm{T}\left(\mathrm{LM}_{\mathrm{T}}\right)$ and create a set $\mathrm{U}=\left\{u_{1}, u_{2}, u_{3}, \ldots, u_{\mathrm{p}}\right\}, u_{\mathrm{j}} \in \mathrm{M}_{\mathrm{b}}$, where the $u_{\mathrm{j}}{ }^{\text {'s }}$ are words that are confused with the word $w$ in $\mathrm{N}_{\mathrm{a}}$ due to speech recognition errors. Again, three cases must be distinguished:

Case B.1.1. There is just one word $\boldsymbol{u}_{\mathbf{j}}$ in $\mathbf{U}$. Let us call this word $u_{1}$. We make ${ }^{5}: \mathrm{D}_{\mathrm{i}} \cdot$ Name $=\mathrm{M}_{\mathrm{b}}, \mathrm{D}_{\mathrm{i}} \cdot w_{\mathrm{i}}=u_{1}, \mathrm{D}_{\mathrm{i}} \cdot c s_{\mathrm{i}}=1.0$, and add $\mathrm{D}_{\mathrm{i}}$ to $e s s p_{\mathrm{BEST}}$.

Case B.1.2. $\mathbf{U}$ is empty. We follow a fallback strategy to find in the $\beta$ set (see Section 3.1.4) the $\mathrm{U}$ set. If there is just one word $u_{\mathrm{j}}$ in $\mathrm{U}$, we proceed as in Case B.1.1. If there are several words $u_{\mathrm{j}}$ 's in $\mathrm{U}$, we proceed as in Case B.1.3. If $U$ is empty we do not make any correction, i.e. we make $D_{i}$ $=\mathrm{C}_{\mathrm{i}}$ and add $\mathrm{D}_{\mathrm{i}}$ to essp $_{\mathrm{BEST}}$.

\footnotetext{
${ }^{5}$ We use the notation ' $\mathrm{D}_{\mathrm{i}} \cdot N a m e$ ', ' $\mathrm{D}_{\mathrm{i}} \cdot w_{\mathrm{i}}$ ' and ' $\mathrm{D}_{\mathrm{i}} \cdot c s_{\mathrm{i}}$ ' to refer to the fields Name, $w_{\mathrm{i}}$ and $c s_{\mathrm{i}}$ of the container $\mathrm{D}_{\mathrm{i}}$, respectively.
} 
Case B.1.3. There are several words $\boldsymbol{u}_{\mathbf{j}}$ in $\mathbf{U}$. We select the $u_{\mathrm{j}}$ with the highest confusion probability $p$, which we call it $u_{\text {high }}$. We make $\mathrm{D}_{\mathrm{i}}$.Name $=\mathrm{M}_{\mathrm{b}}, \mathrm{D}_{\mathrm{i}} \cdot w_{\mathrm{i}}=u_{h i g h}$ and $\mathrm{D}_{\mathrm{i}} \cdot c s_{\mathrm{i}}=p$, and add $\mathrm{D}_{\mathrm{i}}$ to $e s s p_{\mathrm{BEST}}$.

Case B.2. $\mathbf{N}_{\mathbf{a}}=\mathbf{M}_{\mathbf{b}}$. This is the case when the concept obtained from the speech recognition result is assumed to be correct. Hence, we do not change it. We simply make $\mathrm{D}_{\mathrm{i}}=\mathrm{C}_{\mathrm{i}}$ and add $\mathrm{D}_{\mathrm{i}}$ to $e s s p_{\mathrm{BEST}}$.

Case B.3. $\mathbf{N}_{\mathbf{a}}$ cannot be aligned. This is the case when the concept obtained from the speech recognition result is a consequence of an inserted word. To correct the error we discard $\mathrm{C}_{\mathrm{i}}$, i.e. we do not add it to essp $p_{\mathrm{BEST}}$.

\subsubsection{Correction at the linguistic level}

Up to this point we have created $e s s p_{\mathrm{BEST}}$. The correction now aims to correct errors not detected at the statistical level that affect the semantics of the sentences. For example, we have observed in the experiments that when the system prompts to enter product orders, the utterance "una cerveza grande" (one large beer) is sometimes recognised as "dos cerveza grande" (two large beer), which causes the system to incorrectly understand the order as "two large beers". This kind of problem is not detected at the statistical level as the ssp obtained from the incorrect recognition result:

$s s p_{\text {INPUT }}=$ NUMBER SIZE DRINK

matches one of the $s s p$ 's in $\mathrm{SSM}_{\mathrm{T}}$. Hence, $s s p_{\text {INPUT }}$ is not corrected at the statistical level, which results in the essp $p_{\mathrm{BEST}}$ obtained so far being incorrect. To solve this problem we use the set of grammatical rules $\mathrm{R}$ discussed in Section 3.1.2. We place in a window each syntactic-semantic pattern $s s p_{\mathrm{i}}$ in a rule $r_{\mathrm{i}}$. The window slides over $e s s p_{\text {BEST }}$ from left to right. For example, Fig. 5 shows a sample window for the rule $r_{1}$ discussed in Section 3.1.2. 


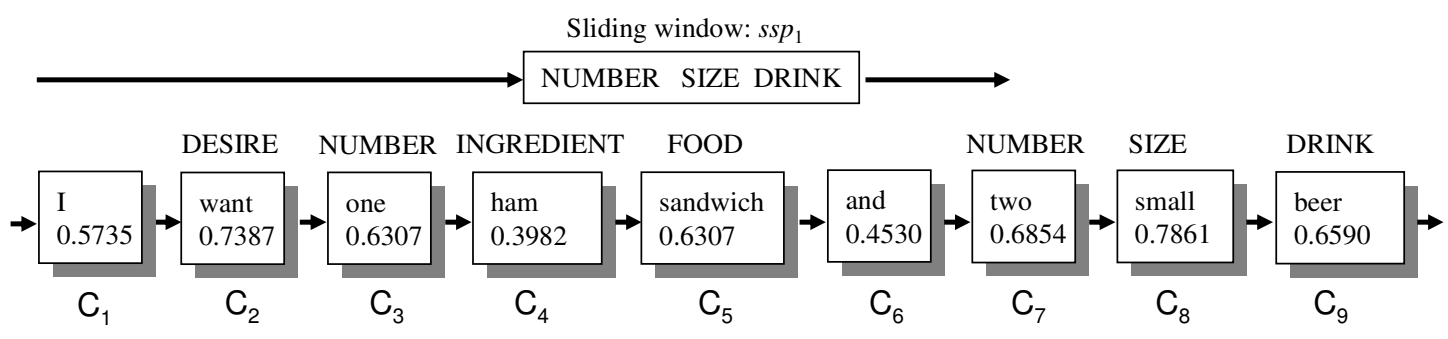

Fig. 5. Window sliding over $e s s p_{\mathrm{BEST}}$.

If the sequence of concepts in the window is found in $\operatorname{essp}_{\mathrm{BEST}}$, restriction $\mathrm{i}$ applies to the words in the containers. For example, in Fig. 5 the sequence of concepts is found in the sequence of containers $\mathrm{C}_{7}-\mathrm{C}_{9}$. Therefore, restriction 1 applies to the words: 'two', 'small' and 'beer'. If the conditions in restriction ${ }_{1}$ are satisfied we do not make any correction, otherwise we try to find the reason for the incongruity by searching for an incorrect word. This is the case in the example given that number(NUMBER) $\neq$ number(DRINK). To find an incorrect word we use the linguistic information available by means of the grammatical rules. In our experiments this is information about the number feature of some Spanish words, for example: number('dos') = plural, number('cerveza') = singular, and number $($ 'pequeña' $)=$ singular $(\operatorname{dos}=$ two, cerveza $=$ beer, pequeña $=$ small $)$. By comparing the number features of these words, we assume that the word 'dos' (two) in container $\mathrm{C}_{7}$ is incorrect, as the number correspondence between 'dos' (two) and 'cerveza' (beer) is incorrect. Hence we define IncorrectContainer $=\mathrm{C}_{7}$ and proceed similarly as explained in Section 3.2.1, Step 2, Case B.1. However, now the goal is not to replace a concept with another concept, but a word within a concept with another word within the same concept. To do this replacement we use the lexical model $\mathrm{LM}_{\mathrm{T}}$ and define the set $\mathrm{U}=\left\{u_{1}, u_{2}, u_{3}, \ldots, u_{\mathrm{p}}\right\}$, where all the words $u_{\mathrm{j}}$ 's belong to the same word-class as the word to be replaced (i.e. NUMBER in the example), are confused with 
this word and satisfy restriction. We finally consider the three cases distinguished in Section 3.2.1, Step 2, Case B.1, and proceed as discussed there.

\subsubsection{Analysis of the performance of the technique for word insertions,}

\section{substitutions and deletions}

Regarding word insertions, the correction is successful if the technique discards the concepts that appear in $s s p_{\text {INPUT }}$ due to inserted words if these words are keywords. The correction can be observed in the following example taken from our experiments, where $\mathrm{T}=$ the prompt type of the dialogue system, $\mathrm{U}=$ the sentence uttered by the user, $h=$ the speech recognition result, and $h^{\prime}=$ the result of the ASRPC module:

T = FOOD_ORDER_CONFIRMATION

$\mathrm{U}=$ one curry salad

$h=$ one $(0.8982)$ error $(0.6950)$ curry $(0.5982)$ salad $(0.8059)$

$h^{\prime}=$ one $(0.8982)$ curry $(0.5982)$ salad $(0.8059)$

The inserted word 'error' caused the concept ERROR to be in the obtained syntacticsemantic pattern: $s s p_{\text {INPUT }}=$ NUMBER ERROR INGREDIENT FOOD. The technique selects the learnt pattern: NUMBER INGREDIENT FOOD as the most similar pattern to $s s p_{\text {INPUT, }}$ and uses it for correction, discarding the concept ERROR at the pattern alignment step.

Regarding word substitutions, the correction requires on the one hand that the technique correctly replaces the incorrect concepts that appear in $s s p_{\text {INPUT }}$ because of the substituted words (assuming that the words are keywords). The replacement can be observed in the following example:

$T=$ TELEPHONE_NUMBER

$U=$ nine one two three four five six seven eight

$h=$ gimme $(0.4982)$ one $(0.6950)$ two $(0.5982)$ three $(0.6059)$ four $(0.8691)$ five $(0.6892)$ six (0.5723) seven (0.5211) eight $(0.8561)$

$h^{\prime}=$ nine $(1.0000)$ one $(0.6950)$ two $(0.5982)$ three $(0.6059)$ four $(0.8691)$ five $(0.6892)$ six (0.5723) seven (0.5211) eight $(0.8561)$

We can see that the uttered word 'nine' was substituted by the word 'gimme'. Hence, $s s p_{\text {INPUT }}=$ DESIRE NUMBER NUMBER NUMBER NUMBER NUMBER NUMBER NUMBER 


\section{ACCEPTED MANUSCRIPT}

NUMBER. The technique selects the learnt pattern: NUMBER NUMBER NUMBER NUMBER NUMBER NUMBER NUMBER NUMBER NUMBER as the most similar learnt pattern to $s s p_{\text {INPUT. }}$ At the pattern matching step it replaces the concept DESIRE with the concept NUMBER, thus correcting the incorrect concept. On the other hand, the correction requires that the technique finds the appropriate candidate word to replace the incorrect word. This is the case in the example, as the technique searches in the word-class NUMBER for a candidate for the word 'gimme' and finds the word 'nine', which is the word uttered by the user.

If the error substitutes a word with another word and both words are keywords in the same word-class, there is no conceptual correction. This is the case, for example, if the user utters the sentence "two ham sandwiches" and it is recognised as "one ham sandwiches", where 'two' and 'one' are keywords in the word-class NUMBER. In this case the correction is successful only if it is successful at the linguistic level.

There is no conceptual correction if the error substitutes a non-keyword with another non-keyword. This happens, for example, if the utterance "please two ham sandwiches and two beers" is recognised as "uhm two ham sandwiches uhm two beers". The conceptual correction fails as there is no change in the sequence of concepts obtained from the utterance.

The technique cannot correct word deletion errors. By carrying out a comparison with the learnt patterns, it can detect that one or more concepts are missing in $s s p_{\text {INPUT }}$ because of the deletion, but it cannot decide the words to fill in the gaps because there are no words that can be used as candidates for the search (given that these words have been deleted). This problem can be observed in the following example:

$T=$ TELEPHONE_NUMBER

$U=$ nine five eight three two zero three one seven 
$h=$ nine $(0.6450)$ five $(0.7941)$ eight $(0.6019)$ three $(0.4002)$ zero $(0.4735)$ three $(0.8998)$ one $(0.8647)$ seven $(0.6953)$

$h^{\prime}=$ nine $(0.6450)$ five $(0.7941)$ eight $(0.6019)$ three $(0.4002)$ zero $(0.4735)$ three $(0.8998)$ one (0.8647) seven (0.6953)

We can see that the uttered word 'two' was deleted. Hence, $s s p_{\text {INPUT }}=$ NUMBER NUMBER NUMBER NUMBER NUMBER NUMBER NUMBER NUMBER. The technique selects the pattern: NUMBER NUMBER NUMBER NUMBER NUMBER NUMBER NUMBER NUMBER NUMBER as the most similar learnt pattern to $s s p_{\text {INPUT. }}$ At the pattern matching step, the technique aligns all the concepts in $s s p_{\text {INPUT }}$ with those in the most similar pattern. The missing concept in $s s p_{\text {INPUT }}$ because of the deleted word has no effect on the matching, and thus there is no correction.

\subsubsection{Advantages of the technique}

The technique requires only a small effort in corpus development if the dialogue corpus needed for training can be easily collected. This is the case when the dialogue system is running in a commercial application or in a research environment, as in these settings it is usually possible to automatically collect a corpus. If the system is not running in any of these settings, we must collect the corpus from the start, or use a corpus that is already available for the same application domain, perhaps collected using another dialogue system. Hence, this satisfies only partly one of our initial goals when developing the technique, i.e. minimal initial effort in corpus development.

The effort for assigning types $(\mathrm{T})$ to the prompts generated by a dialogue system is very small, which satisfies one of our initial goals when developing the technique: easy application to different tasks. Using grammatical rules instead of statistical information for the correction at the linguistic level, satisfies another of our initial goals: minimal requirements for training data. The simple algorithms discussed in Section 3.2 


\section{ACCEPTED MANUSCRIPT}

make it easy to set up the technique, which satisfies another initial goal: easy implementation.

The technique learns very rapidly from the training data. This happens because the syntactic-semantic structure of the sentences used for training is represented by means of patterns comprised of word-classes. This kind of pattern allows us to generalise knowledge obtained from the training, and to apply it to cases not observed in the training (Ward and Issar, 1996).

The technique is robust against some spontaneous speech phenomena. For example, it can handle hesitations (e.g. 'uhm') typically uttered by users when they are thinking what to say next in the dialogue. This happens because hesitations are not keywords, and thus they do not affect the sequence of concepts obtained from the analysis of the sentence. For example, if the user utters either "uhm ... one ham sandwich" or "one ham sandwich", $s s p_{\text {INPUT }}$ is: NUMBER INGREDIENT FOOD.

The technique is also robust against repeated words typically uttered in spontaneous speech, provided that this phenomenon is observed in the training dialogue corpus. For example, if the sentence "one ... uhm ... one ... ham sandwich" is in this corpus, the technique learns the syntactic-semantic pattern: $s s p=$ NUMBER NUMBER INGREDIENT FOOD. In this way, if a user utters the sentence "two ... uhm ... two vegetarian salads please", $s s p_{\text {INPUT }}$ is: NUMBER NUMBER INGREDIENT FOOD, and thus the technique does not make any correction at the pattern matching step. Note that this pattern is also useful for the changes of mind typical of spontaneous speech, where the user corrects data as he speaks. This phenomenon can be observed in the following example: "one ... uhm ... well ... uhm ... two ham sandwiches please". For this sentence, 
$s s p_{\text {INPUT }}$ is also: NUMBER NUMBER INGREDIENT FOOD, and thus the technique does not make any correction at the pattern matching step.

The effect of partially uttered words, also typical of spontaneous speech, depends on the kind of speech recognition error they cause. If by chance these words cause a kind of error observed in the training, the error may be corrected. For example, let us suppose that the user utters the sentence "I would like one sm ... small beer please", where he partially utters the word 'small'. If the recognition result is e.g. "I would like one is small beer please" the error can be corrected as $s s p_{\text {INPUT }}$ would be: DESIRE NUMBER SIZE DRINK, which is observed in the training. The technique would discard the word 'is' as it is not a keyword. The technique would also be successful if the recognition result was: "I would like one s small beer please". For this input $s s p_{\text {INPUT }}$ would be: DESIRE NUMBER LETTER SIZE DRINK, given that the word 's' is the LETTER word-class. As the most similar learnt pattern to the input pattern would be: DESIRE NUMBER SIZE DRINK, the result of the pattern alignment would be the removal of the LETTER concept, and thus the error would be corrected as the word 's' would be discarded.

It is possible to think of cases where the technique would fail when dealing with partially uttered words. For example, the user could utter the sentence "one ve ... uhm one green salad please" where he changes his mind, leaving the word 'vegetarian' partially uttered. The recognition result for this utterance could be e.g. "one beer and one green salad please" and thus $s s p_{\text {INPUT }}$ would be: NUMBER DRINK NUMBER INGREDIENT FOOD. As this pattern is observed in the training, there would be no correction at the pattern matching step and the error would remain uncorrected. 


\section{Experiments}

The goal of the experiments was to test the effect of the proposed technique on the performance of the Saplen system. To do this we compared evaluation results obtained with two speech recognition front-ends:

i) The standard HTK-based speech recogniser of the Saplen system (baseline).

ii) The enhanced speech recogniser shown in Fig. 6, which employs the ASRPC (ASR Post-Correction) module that implements the proposed technique.

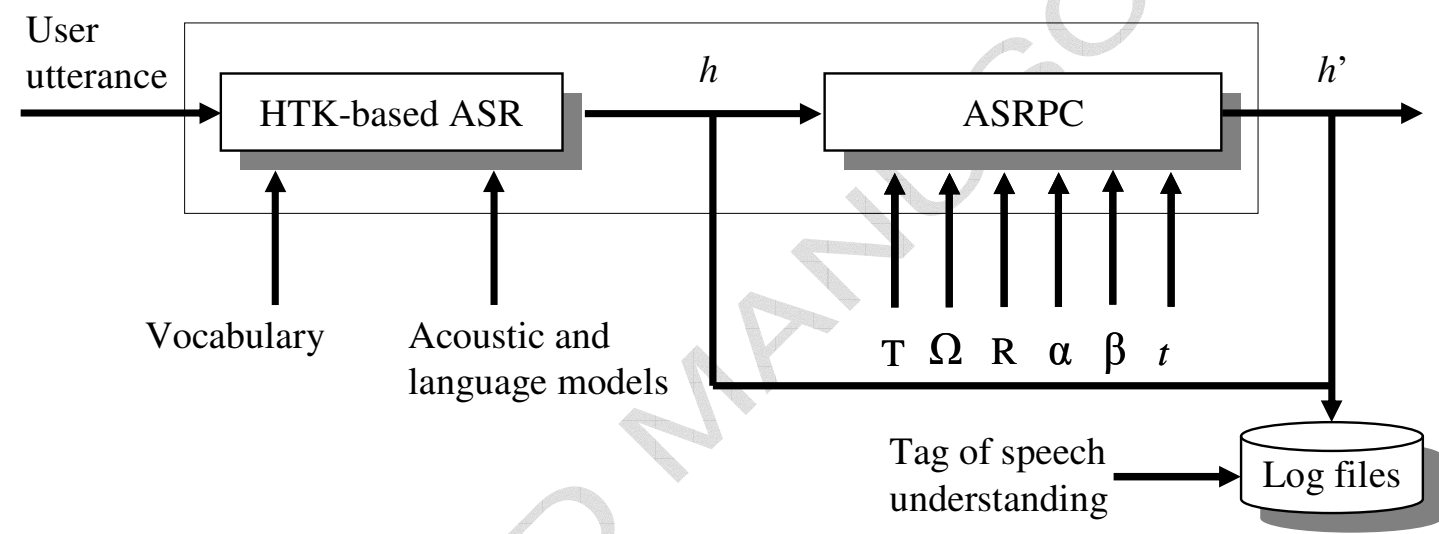

Fig. 6. Enhanced speech recogniser used in the experiments.

In the figure $\mathrm{T}$ denotes the current prompt type of the dialogue system, $\Omega$ represents the set of word-classes, $\mathrm{R}$ is the set of grammatical rules, $\alpha$ is the set of syntactic-semantic models, $\beta$ is the set of lexical models, and $t$ is the similarity threshold. The input to the ASRPC module was a recognition result provided by the speech recogniser: $h=w_{1} c s_{1}$ $w_{2} c s_{2} \ldots w_{\mathrm{n}} c s_{\mathrm{n}}$, where the $w_{\mathrm{i}}$ 's represent words and the $c s_{\mathrm{i}}$ 's confidence scores. The output of the module was another speech recognition result: $h^{\prime}=w^{\prime}{ }_{1} c s^{\prime}{ }_{1} w^{\prime}{ }_{2} c s_{2}^{\prime} \ldots w_{\mathrm{m}}^{\prime}$ $c s_{\mathrm{m}}$, where some words and confidence scores may be changed.

The performance of the HTK-based recogniser and the ASRPC module was saved in log files for evaluation purposes. Each entry in these files contained the 
transcription of each utterance, the speech recognition result $(h)$, the result of the ASRPC module ${ }^{6}\left(h^{\prime}\right)$, and a tag indicating whether the speech recognition result was correctly understood by the Saplen system.

\subsection{Evaluation measures}

Experimental results were obtained in terms of word accuracy (WA), speech understanding (SU), implicit recovery (IR) and task completion (TC) (Danieli and Gerbino, 1995). WA is the proportion of correctly recognised words. It was computed as the percentage WA $=\left(w_{t}-w_{i}-w_{s}-w_{d}\right) \times 100 / w_{t}$, where $w_{t}$ is the total number of words in the analysed sentences, and $w_{i}, w_{s}$ and $w_{d}$ are the numbers of words inserted, substituted and deleted by the speech recogniser of the Saplen system, respectively.

Sentence understanding (SU) is the proportion of sentences correctly understood by the Saplen system as it interacted with the user simulator. It was computed as the percentage $S U=S_{u} \times 100 / S_{t}$, where $S_{u}$ is the number of analysed sentences where the obtained semantic representation was completely correct (i.e. it was exactly the same as the correct semantic representation) and $\mathrm{S}_{\mathrm{t}}$ is the total number of sentences analysed.

The semantic analyser of the Saplen system is robust against some kinds of recognition error, which enabled it to correctly understand some sentences even though some words were misrecognised. Implicit recovery (IR) is the proportion of incorrectly recognised sentences that were correctly understood by the dialogue system. It was computed as the percentage IR $=S_{u} \times 100 / S_{e}$, where $S_{u}$ is the number of analysed sentences in which the obtained semantic representation was completely correct, and $\mathrm{S}_{\mathrm{e}}$ is the number of incorrectly recognised sentences.

\footnotetext{
${ }^{6}$ This data was stored only when the ASRPC module was used.
} 
Task completion (TC) is the proportion of successful dialogues, i.e. the percentage of dialogues that ended with all the scenario goals achieved. It was computed as the percentage $T C=D_{c} \times 100 / D_{t}$, where $D_{c}$ is the number of successful dialogues and $D_{t}$ is the total number of dialogues. As will be discussed in the following section, the user simulator cancelled the interaction with the dialogue system if the dialogue became too long due to system malfunction. Cancelled dialogues were not considered successful and thus decreased the TC rate.

\subsection{Interaction between the Saplen system and the user simulator}

The Saplen system was developed in a previous study to answer Spanish telephonebased orders and queries by clients of fast food restaurants (López-Cózar et al. 1997). To develop the system we collected a dialogue corpus in a fast food restaurant that contains about 800 recorded dialogues in Spanish involving conversations between clients and restaurant assistants (López-Cózar et al. 1998). These dialogues contain product orders, telephone numbers, postal codes, addresses, queries, confirmations, greetings and other types of sentence. The dialogues were transcribed, labelled and analysed to include tags regarding the speakers (clients and restaurant assistants), sentence types, semantic information of sentences and other kinds of information. From this corpus we created the Saplen corpus which contains 5,500 client utterances and about 2,000 different words, as well as the transcriptions and semantic representations of the utterances stored as frames (Allen, 1995). We have used this corpus for previous studies (e.g. López-Cózar et al. 2003; López-Cózar and Callejas, 2005).

In a previous study we developed a user simulator the purpose of which is to interact automatically with a dialogue system to obtain a dialogue corpus suitable for testing purposes (López-Cózar et al. 2003). The most recent version of the simulator 
supports three different types of user: very cooperative, cooperative and not very cooperative, in order to simulate a wider variety of user inputs (López-Cózar et al. 2007). When simulating a very cooperative user, the simulator always provides the type of data the system asks for, and when the system prompts to confirm data, it always answers an appropriate affirmative or a negative confirmation. When simulating a cooperative user, the simulator always provides the type of data the system asks for, and when the system prompts to confirm data, it sometimes answers an appropriate affirmative or a negative confirmation, and in other cases it repeats the data being confirmed. When simulating a not very cooperative user, the simulator selects randomly the type of data it provides when the dialogue system asks for a particular data, and when the system prompts to confirm data it behaves as a cooperative user. Fig. 7 shows the connection between the user simulator and the Saplen system.

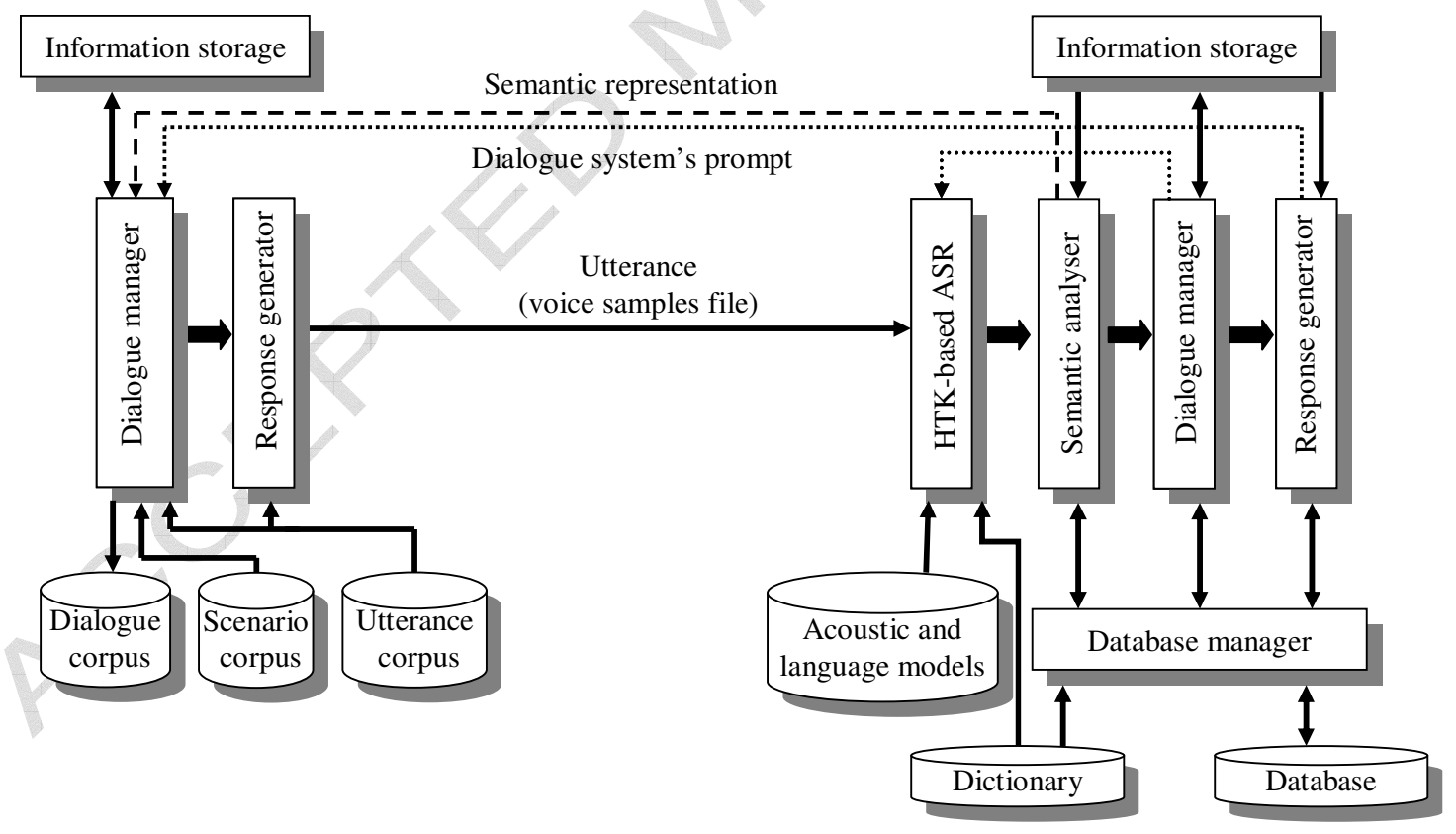

Fig. 7. The user simulator (on the left) interacting with the Saplen system (on the right). 


\section{ACCEPTED MANUSCRIPT}

The user simulator receives the current prompt generated by the dialogue system as well as the semantic representation obtained by the system from the analysis of the previous simulator's response. Therefore, the semantic representation can be affected by recognition and understanding errors similarly as if the simulator response had been uttered by a real user. To interact with the dialogue system the user simulator employs a set of scenarios that indicate the goals it must try to achieve during the interaction. For example, the scenario shown in Fig. 8 indicates that the simulator must order initially "one large cola" and "one ham sandwich". When the system prompts for additions to the order, it must order "one green salad". The scenario goals are semantic representations (frames) of utterances in the corpus.

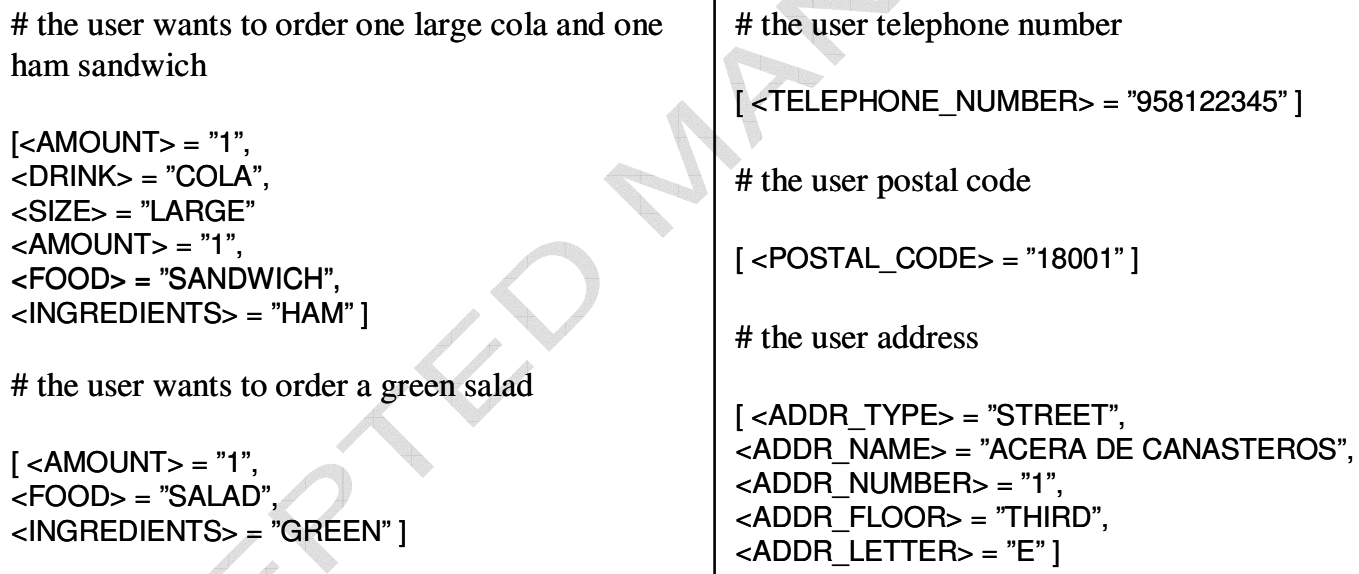

\# the user telephone number

[ $<$ TELEPHONE_NUMBER> = "958122345"]

\# the user postal code

[ <POSTAL_CODE $>=$ "18001"]

\# the user address

[ $<$ ADDR_TYPE $>=$ "STREET",

$\angle A D D R$ NAME $>=$ "ACERA DE CANASTEROS", $<A D D R \_N U M B E R>=" 1 "$, $<$ ADDR_FLOOR $>=$ "THIRD", $<A D D R \_L E T T E R>=$ "E" ]

Fig. 8. A sample scenario for the user simulator.

When the system prompts for the user's telephone number, postal code or address, the user simulator employs the telephone number, postal code and address in the scenario. When the appropriate scenario goal, e.g. $\angle$ POSTAL_CODE $>=" 18001 "$, is selected to 
answer a system prompt, the simulator selects at random ${ }^{7}$ any utterance in the corpus (voice samples file) the semantic representation of which matches the selected goal, for example "my postal code is 18001 ". This utterance is the input for the dialogue system's HTK-based speech recogniser, as can be observed in Fig. 7. The dialogues generated in the system-simulator interaction are stored in $\log$ files for evaluation purposes, including the system prompt, the prompt type (T), the user simulator's response (utterance transcription) and the speech recognition result. The simulator employs an interactionLimit parameter to cancel the dialogue with the dialogue system if the total number of interactions (i.e. of system plus user simulator) exceeds a threshold set to 30 interactions. The value of this parameter was decided considering the complexity of the scenarios employed for the interaction. Below is a sample dialogue between the system and the user simulator behaving as a not very cooperative user.

(1) Saplen: Good evening, welcome to our restaurant. This is our automatic telephone service system. We inform you that the minimum price for the home delivery service is six euros. Please speak after the tone. What would you like to have?

T = ANYTHING_TO_ORDER

(2) User simulator: Acera de Canasteros Street, number one, third floor, letter e [Recognition result: cherry (1.0000) make (0.5997) an (0.0718) order (0.718) of (0.6070) elm (0.4084)]

(3) Saplen: I'm sorry, I didn't understand. You can order products or ask for information. How may I help you? T = ORDER_OR_INFORMATION

(4) User simulator: one large cola and one ham sandwich [Recognition result: one (0.7991) large $(0.9785)$ cola $(0.4997)$ and $(0.4871)$ one $(0.4942)$ ham $(0.6591)$ sandwich $(0.6781)]$

(5) Saplen: OK. One large cola and one ham sandwich. Would you like anything else? T = ANYTHING_TO_ORDER

(6) User simulator: one green salad [Recognition result: one (0.8564) green (0.8564) salad (0.5864)]

(7) Saplen: OK. Please say your telephone number. T = TELEPHONE_NUMBER

(8) User simulator: 958122345 [Recognition result: eighteen (1.0000) zero (0.8354) zero (0.6923) seven (0.4999) forty-five (0.3870)]

(9) Saplen: I need to confirm your telephone number. Did you say $18,0,0,7,45$ ? $T=$ TELEPHONE_CONFIRMATION

\footnotetext{
${ }^{7}$ To employ all the utterances available in the corpus, the simulator first selects utterances that have not been used. When all have been used, it selects those least used, which helps to employ all the possible utterances approximately the same number of times.
} 
(10)User simulator: 958122345 [Recognition result: seventy-eight (0.5025) twelve (0.7002) seventy-five (0.6698)]

(11)Saplen: OK. Telephone number 78, 12, 75. Please say your postal code $\mathrm{T}=$ POSTAL_CODE

(12)User simulator: This is incorrect [Recognition result: this $(0.4048)$ is $(0.6992)$ incorrect (1.0000)]

(13)Saplen: OK. Please say your telephone number again. $\mathrm{T}=$ TELEPHONE_NUMBER

(14)User simulator: 958122345 [Recognition result: nine (0.9390) five (0.9581) eight (0.3919) twelve (0.6750) twenty-three $(0.7848)$ forty-five $(0.9816)]$

(15)Saplen: I must confirm your telephone number again. Did you say 9, 5, 8, 12, 23, 45? $T=$ TELEPHONE_CONFIRMATION

(16)User simulator: 958122345 [Recognition result: nine (0.7835) five (0.8273) eight (0.8471) twelve (0.9849) twenty-three $(0.6084)$ forty-five $(0.8481)]$

(17)Saplen: OK. Telephone number 9, 5, 8, 12, 23, 45. Please say your postal code. $\mathrm{T}=\mathrm{POSTAL}$ CODE

(18)User simulator: 18001 [Recognition result: eighteen (0.7984) zero (1.0000) zero (1.0000) one (0.5048)]

(19)Saplen: OK. Postal code number 18, 0, 0, 1. Please say your address, for example, Elm street, number 13 , first floor, letter $\mathrm{e}$. $T=$ ADDRESS

(20)User simulator: Acera de Canasteros street, number one, third floor, letter e [Recognition result: acera-de-canasteros (1.0000) street (1.000) ice (0.3046) error (0.3046) twelve (1.0000) beer (0.1862)]

(21)Saplen: I have to confirm the building number. I think you said 12. Is this correct? $T=$ BUILDING_NUMBER

(22)User simulator: one [Recognition result: two (0.4223)]

(23)Saplen: I have to confirm the building number again. Did you say 2? T = BUILDING_NUMBER_CONFIRMATION

(24)User simulator: one [Recognition result: one (0.5891)]

(25)Saplen: OK. Number one. Please say the floor of the apartment. T = APARTMENT_FLOOR

(26)User simulator: third [Recognition result: first (0.8012)]

(27)Saplen: OK. First floor. Please say the apartment letter. $\mathrm{T}=$ APARTMENT_LETTER

(28) User simulator: This is incorrect [Recognition result: this (0.5091) is (0.9892) incorrect (0.7432]

(29)Saplen: OK. Please say floor again. T = APARTMENT_FLOOR

*** INTERACTION LIMIT (30) REACHED, DIALOGUE CANCELLED BY USER SIMULATOR ***

Employing this kind of dialogue we aim to simulate real dialogues in which the users sometimes utter sentences that do not match the current prompt of the dialogue system, as can be observed in the following interaction:

(1) Human operator: Good evening, welcome to our restaurant. What would you like to have?

(2) User: one ham sandwich and one large beer please

(3) Human operator: Anything else?

(4) User: no, thanks.

(5) Human operator: What is your telephone number?

(6) User: well, uhm ... I think l'd better have one small beer instead of one large beer

(7) Human operator: OK, small beer. Telephone number, please? 
Turn (6) of this example would not be recognised if at this dialogue state the system used a prompt-dependent language model trained from telephone numbers only. Hence, it is important to use wide coverage grammars that allow the system, in principle, to recognise any kind of sentence permitted in the application domain, regardless of the current prompt of the dialogue system. The problem is that these grammars are more complex, and the vocabulary active at each dialogue state is much larger, which tends to markedly increase speech recognition errors.

\subsection{The speech database and test scenarios for the user simulator}

We used two separate utterance corpora, one for training and the other for testing, that we employed in a previous study (López-Cózar et al. 2003). Both disjunct corpora were created using the Saplen corpus discussed in the previous section, selecting utterances at random among the 18 types shown in Table 4 . Each corpus included the transcriptions of the utterances as well as their corresponding semantic representations.

Table 4. Utterance corpora used for training and testing.

\begin{tabular}{lcc}
\hline Sentence type & No. training utterances & No. test utterances \\
\hline Product order & 250 & 250 \\
Telephone number & 250 & 250 \\
Postal code & 250 & 250 \\
Address & 250 & 250 \\
Query & 125 & 125 \\
Confirmation & 125 & 125 \\
Number & 125 & 125 \\
Food name & 125 & 125 \\
Ingredient & 125 & 125 \\
Drink name & 125 & 125 \\
Size & 125 & 125 \\
Taste & 125 & 125 \\
Temperature & 125 & 125 \\
Street name & 125 & 125 \\
Building number & 125 & 125 \\
Building floor & 125 & 125 \\
Apartment letter & 125 & 125 \\
Error indication & 125 & 125 \\
\hline & Total: 2,750 & Total: 2,750 \\
\hline
\end{tabular}


We compiled one prompt-independent language model (word bigram) from the 2,750 training sentences to enable the recognition of the 18 sentence types set out in Table 4 . In theory, this language model would provide users with a more natural interaction as they could utter any of the 18 sentence types at any moment in the dialogue, regardless of the current prompt generated by the system. However, we observed in previous experiments that using such a grammar degraded the word accuracy given that the vocabulary was large and there were many possible sentences to be considered during the analysis of each utterance. This problem motivated the proposed technique, which is intended to increase word accuracy when such a language model is used.

Employing the test utterance corpus we automatically created 400 different scenarios similar to that shown in Fig. 8. To do this we used the semantic representations of the 250 product orders, telephone numbers, postal codes and addresses in this corpus. Each scenario contained one product order, which was created by means of the random combination of $1-3$ semantic representations of product orders. The scenario also contained the semantic representation of one telephone number, one postal code and one address, also selected at random. To carry out the experiments the scenario corpus was divided into two separate scenario corpora, which we called ScenariosA (300 scenarios) and ScenariosB (100 scenarios).

\subsection{Experiments to decide the requirements on training corpus}

We initially carried out additional experiments to decide the appropriate number of dialogues that enabled the syntactic-semantic and lexical models to obtain the maximum amount of information from the training. We employed the Saplen system and the user simulator and increased the number of automatically generated dialogues until we did not observe any change in the amount of learnt information, nor in terms of syntactic- 
semantic patterns in the $\alpha$ set, nor in terms of lexical confusions in the $\beta$ set. Table 5 shows the results obtained.

Table 5. Progress of learnt information as the size of training corpus increases.

\begin{tabular}{ccc}
\hline Number of dialogues & Number of patterns in $\alpha$ & Number of lexical confusions in $\beta$ \\
\hline 150 & 146 & 408 \\
300 & 161 & 551 \\
450 & 167 & 684 \\
600 & 172 & 763 \\
750 & 175 & 908 \\
900 & 175 & 1,042 \\
1050 & 175 & 1,042 \\
\hline
\end{tabular}

It can be observed that the number of syntactic-semantic patterns and lexical confusions increased with the number of generated dialogues until it reached a threshold. The maximum number of patterns (175) was obtained for 750 generated dialogues, whereas the highest number of lexical confusions $(1,042)$ was achieved for 900 dialogues. Hence, we assumed that 900 was the optimum number of dialogues that needed to be generated.

\subsection{Experiments with the baseline system}

In these experiments we employed the original HTK-based speech recogniser of the Saplen system only. Hence, the recognition results that were the input to the semantic analyser of the system were not corrected by the ASRPC module. Employing ScenariosA and the user simulator we generated a corpus of 900 dialogues that we called Dialogues $A_{1}$. To create these dialogues the simulator used each scenario 3 times, behaving as a very cooperative user, a cooperative user, and a not very cooperative user. Table 6 sets out the average results obtained from the analysis of this dialogue corpus.

Table 6. Average evaluation results (in \%) of the baseline system.

\begin{tabular}{lcccc}
\hline Dialogue corpus & WA & SU & IR & TC \\
\hline Dialogues $_{1}$ & 76.12 & 54.71 & 9.19 & 24.51 \\
\hline
\end{tabular}


The low WA rate shows the problems of the speech recogniser in correctly analysing the utterances. These problems affected the other evaluation measures, which were very low. Observing the log files created (see Fig. 6) we found that in some cases the recognition results were incorrect but similar to the input utterances. For example, "una cerveza grande" (one large beer) was recognised as: "una cerveza grandes", which was correctly understood by the system because of its implicit recovery ability. We found that $9.19 \%$ of the incorrectly recognised sentences were correctly understood by the system due to this reason. In other cases, the recognition results were completely different to the utterances and it was impossible for the system to implicitly recover the errors. For example, the address: "calle Acera de Canasteros numero uno, tercero, letra e" (Acerca de Canasteros Street, number one, third foor, letter e) was recognised as: "e de cereza hacer un pedido necesito de bazan" (e of cherry order I need of bazan).

\subsection{Experiments with the proposed technique}

\subsubsection{Creation of the elements for applying the technique}

In accordance with Section 3.1.1, we created a set of word-classes $\Omega=\left\{K_{1}, K_{2}, \ldots\right.$, $\mathrm{K}_{21}$ \} by re-using the 21 word-classes available from a previous study (López-Cózar and Callejas, 2005). These classes were the same word-classes used by the semantic analyser of the Saplen system. According to Section 3.1.2, we created a set of grammatical rules that contained 3 rules to check number correspondences in food and drink orders uttered in Spanish. To create the sets of syntactic-semantic and lexical models, as discussed in Sections 3.1.3 and 3.1.4, we used Dialogues $A_{1}$ and obtained $\alpha=$ $\left\{\mathrm{SSM}_{\mathrm{Ti}}\right\}$ and $\beta=\left\{\mathrm{LM}_{\mathrm{Ti}}\right\}, \mathrm{i}=1 \ldots 43$ (43 is the number of prompt types generated by the Saplen system). 


\subsubsection{Decision on the optimal value for the similarity threshold}

To decide the best value for the similarity threshold $t$, we carried out side experiments testing 6 different values: $0.3,0.4,0.5,0.6,0.7$ and 0.8 . We used ScenariosB (100 dialogues) and employing the user simulator we generated 6 dialogue corpora, one per value of $t$. The user simulator employed each scenario 3 times, simulating very cooperative, cooperative and not very cooperative users. Hence, each corpus contained 300 dialogues. The Saplen system used the ASRPC module during this corpora generation, employing the $\alpha$ and $\beta$ sets created in the previous section. Analysis of the 6 corpora showed that the best performance of the system was achieved when $t=0.5$.

\subsubsection{Using the proposed technique with the optimal value of the similarity threshold}

In these experiments the output of the HTK-based speech recogniser was processed by the ASRPC module (see Fig. 6). The similarity threshold was set to the optimal value: $t$ $=0.5$. We used again ScenariosA (300 scenarios) and employing the user simulator we generated 900 dialogues, which we called Dialogues $A_{2}$. As in the previous experiments, each scenario was used three times, one per user type. Table 7 shows the average results obtained from the analysis of this dialogue corpus.

Table 7. Average evaluation results (in \%) employing the proposed technique.

\begin{tabular}{lcccc}
\hline Dialogue corpus & WA & SU & IR & TC \\
\hline DialoguesA $_{1}$ & 84.62 & 71.25 & 13.20 & 68.32 \\
\hline
\end{tabular}

Analysis of the log files showed that the ASRPC module was successful in correcting some erroneous recognition results. Table 8 shows some examples of these corrections, where $\mathrm{T}=$ prompt type of the Saplen system, $\mathrm{U}=$ user utterance, $h=$ speech recognition 
result, and $h^{\prime}=$ result of the ASRPC module. The corrections were carried out by

replacing or discarding words in $h$.

Table 8. Examples of successful corrections of speech recognition errors (in Spanish).

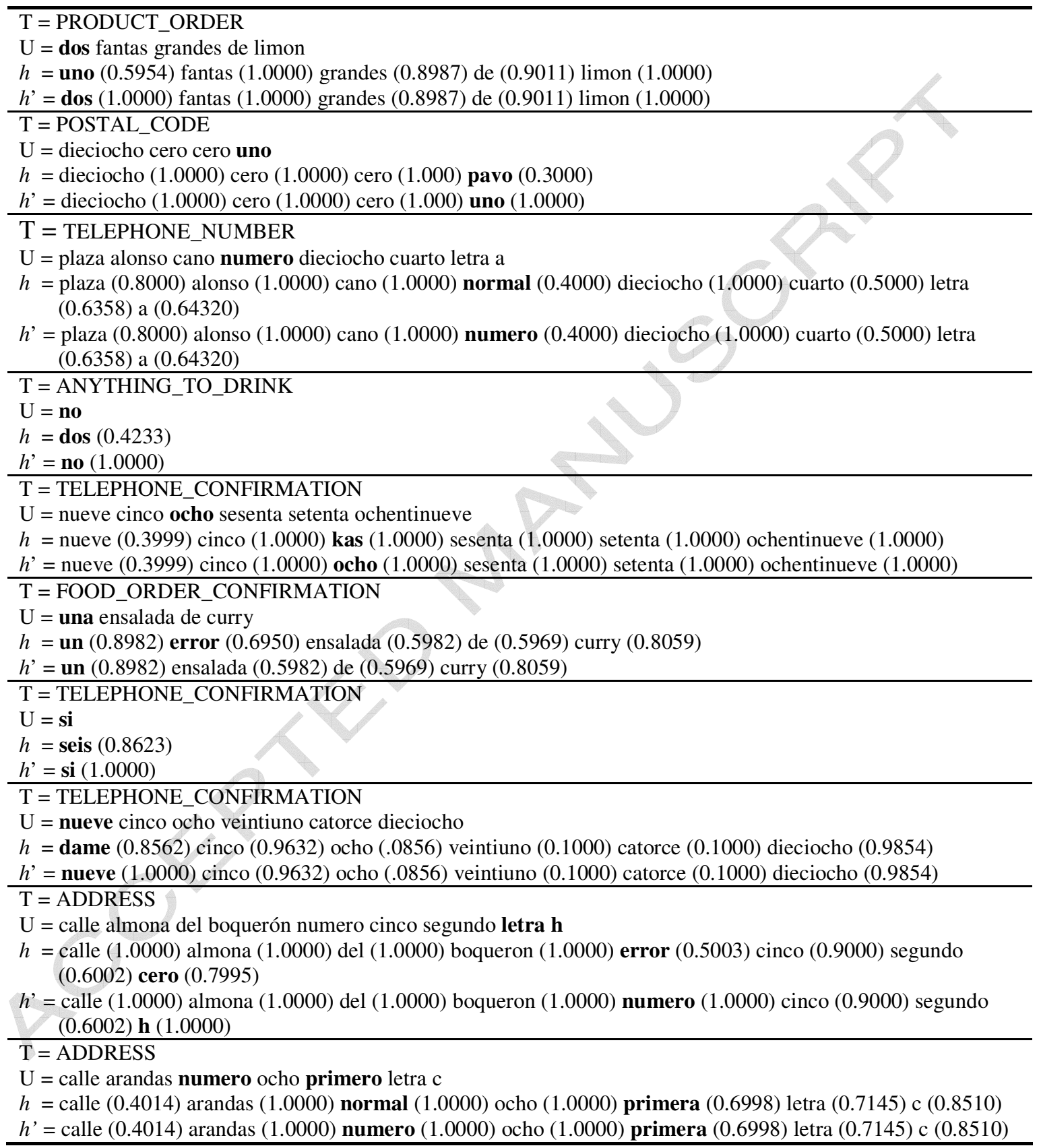

The technique worked very well for correcting errors in affirmative or negative confirmations. This happened because the speech recogniser usually substituted the 
word 'si' (yes) employed in many affirmative confirmations by the word 'seis' (six), especially when 'si' was uttered by speakers with strong southern Spanish accents. These users generally omitted the final 's' of words, thus making 'seis' to be acoustically very similar to 'si'. Also because of these accents, the recogniser usually substituted the word 'no' by the word 'dos'. The ASRPC module corrected both kinds of error by replacing the concept NUMBER with the concept CONFIRMATION, and then selecting the most likely word in the latter concept given the word 'seis' (in affirmative confirmations) or 'dos' (in negative confirmations).

The technique also corrected many incorrectly recognised product orders. For example, "dos fantas grandes de limon" (two large lemon fantas) was recognised as "uno fantas grandes de limon" (one large lemon fantas) because of the acoustic similarity between 'dos' en 'uno' when uttered by strongly accented speakers. The ASRPC module repaired the error doing no corrections at the statistical level, and replacing 'uno' with 'dos' at the linguistic level. In other cases the correction was carried out at the statistical level. For example, "una ensalada de curry" (one curry salad) was recognised as "un error ensalada de curry" (one mistake curry salad). The error correction was carried out by discarding the word in the concept ERROR ('error'). The outcome of the ASRPC ("un ensalada de curry") contained one gender discordance in Spanish as 'un' is male whereas 'ensalada' is female, but the sentence was correctly understood by the Saplen system as the discordance did not affect the semantic content of the sentence.

The technique was also able to correct some misrecognised telephone numbers. For example, "nueve cinco ocho veintiuno catorce dieciocho" (nine five eight twenty-one fourteen eighteen) was recognised as "dame cinco ocho veintiuno catorce dieciocho" (gimme five eight twenty-one fourteen eighteen) because of the acoustic similarity 
between 'nueve' and 'dame'. The ASRPC module corrected the error by replacing the concept DESIRE with the concept NUMBER, and selecting the most likely word in the latter concept ('nueve') given the word 'dame'.

The technique was also useful in repairing some misrecognised postal codes. For example, "dieciocho cero cero uno" (eighteen zero zero one) was recognised as "dieciocho cero cero pavo" (eighteen zero zero turkey). This error was corrected by replacing the concept INGREDIENT with the concept NUMBER, and selecting the most likely word in the latter concept ('uno') given the word 'pavo'.

The ASRPC module was also successful in correcting some incorrectly recognised addresses. For example, "calle almona del boquerón numero cinco segundo letra h" (almona del boqueron street number five second ${ }^{8}$ letter $\mathrm{h}$ ) was recognised as "calle almona del boqueron error cinco segundo cero" (almona del boqueron street error five second zero). The error was corrected by making a double repair. First, replacing the concept ERROR with the concept NUMBER_ID, and selecting the most likely word in the latter concept ('numero') given the word 'error'. Second, replacing the concept NUMBER with the concept LETTER, and selecting the most likely word in the latter concept (' $h$ ') given the word 'zero'.

\subsubsection{Advantage of using contextual syntactic-semantic models $\left(\mathrm{SSM}_{\mathrm{T}}\right.$ 's)}

The goal of this experiment was to check whether using different $\operatorname{SSM}_{\mathrm{T}}$ 's, and if needed $\alpha=\left\{\mathrm{SSM}_{\mathrm{Ti}}\right\}$ as a fallback strategy, as discussed in Section 3.2.1, was preferable to the two following alternative strategies:

\footnotetext{
${ }^{8}$ In this sentence in Spanish, the word 'floor' was implicit after the word 'second', i.e. the data for the system was 'second floor'.
} 


\section{ACCEPTED MANUSCRIPT}

i) use $\alpha$ only, without first checking the prompt-dependent $\mathrm{SSM}_{\mathrm{T}}$ 's.

ii) use $\mathrm{SSM}_{\mathrm{T}}$, and if the pattern is not found there use $\alpha$ as a fallback strategy, but without considering ${ }^{9}$ the similarity threshold $t$.

The $\alpha$ set was that created with DialoguesA $A_{1}$ in Section 4.6.3, and the similarity threshold was set to the optimal value, $t=0.5$. We first changed slightly the behaviour of the ASRPC module so that it worked in accordance with the procedure described in i). We used again ScenariosA (300 scenarios) and the user simulator to generate a corpus of 900 dialogues, which we called Dialogues $A_{3}$ (each scenario was used three times, one per user type). Next we changed again the behaviour of the ASRPC module so that it now worked in accordance with the procedure described in ii). We used again ScenariosA and the user simulator and generated another corpus of 900 dialogues, which we called Dialogues $A_{4}$ (each scenario was used three times, one per user type). Therefore, Dialogues $A_{1}$, Dialogues $A_{3}$ and Dialogues $A_{4}$ were created using the same scenarios and were comprised of the same number of dialogues, the only difference being in the strategy for selecting the syntactic-semantic model to be used. Table 9 shows the average results obtained from the analysis of Dialogues $A_{3}$ and Dialogues $A_{4}$.

Table 9. Average evaluation results (in \%) obtained by changing the strategy to select the syntactic-semantic model.

\begin{tabular}{|c|c|c|c|c|}
\hline Dialogue corpus & $\overline{\mathrm{WA}}$ & $\overline{\mathrm{SU}}$ & IR & $\mathrm{TC}$ \\
\hline Dialogues $\mathrm{A}_{3}$ (using $\alpha$ only) & 80.15 & 61.67 & 9.57 & 39.78 \\
\hline DialoguesA $_{4}$ (using $\mathrm{SSM}_{\mathrm{T}}$ if possible, otherwise use $\alpha$ ) & 82.26 & 66.84 & 12.15 & 55.35 \\
\hline
\end{tabular}

\footnotetext{
${ }^{9}$ Note that the proposed strategy takes into account the similarity threshold $t$. The fallback strategy, i.e. use of the $\alpha$ set, is employed only if no patterns are found in $\mathrm{SSM}_{\mathrm{T}}$ similar to the input pattern with similarity value greater than $t$.
} 
Analysis of the $\log$ files showed that the error correction in confirmations was very much affected by the strategy employed to select the correction model (either $\mathrm{SSM}_{\mathrm{T}}$ or $\alpha$ ). This selection had a considerable effect on dialogue success because correctly recognising confirmations is critical for the dialogue. If we always used $\mathrm{SSM}_{\mathrm{T}}$ to correct errors in confirmations, the correction was in many cases successful. On the other hand, if we always used $\alpha$ the correction was mostly incorrect. This happened because the pattern comprised of just of the concept NUMBER (i.e. $s s p=$ NUMBER) was in $\alpha$. Therefore, if the answer to a confirmation prompt was 'si' (yes) and it was incorrectly recognised as 'seis' (six), the input pattern obtained from the recognition result was: ssp $=$ NUMBER, which matched one pattern in $\alpha$. Hence, the algorithm for error correction did not make any correction at the pattern matching step and the recognition result remained uncorrected. If we compare Table 9 and Table 7 (Section 4.6.3) it follows that the best strategy for selecting the model for the conceptual correction (either $\mathrm{SSM}_{\mathrm{T}}$ or $\alpha$ ) is the one proposed in this paper, which makes the decision considering the similarity threshold.

\subsubsection{Advantage of using contextual lexical models ( $\mathrm{LM}_{\mathrm{T}}$ 's)}

Analogously to the previous section, the goal of this experiment was to check whether using different $\mathrm{LM}_{\mathrm{T}}$ 's and if needed $\beta=\left\{\mathrm{LM}_{\mathrm{Ti}}\right\}$ as a fallback strategy, as discussed in Section 3.2.2, was preferable to using $\beta$ only. To carry out the experiment we used the $\beta$ set created with Dialogues $A_{1}$ in Section 4.6.3. Similarly as we did in the previous section, we changed slightly the behaviour of the ASRPC module in accordance with

the new strategy, i.e. use $\beta$ always instead of different $\mathrm{LM}_{\mathrm{T}}$ 's. We employed again ScenariosA (300 scenarios) and the user simulator and generated a corpus of 900 dialogues, which we called Dialogues $A_{5}$ (each scenario was used three times, one per 
user type). Therefore, Dialogues $A_{1}$ and Dialogues $A_{5}$ were obtained using the same scenarios and were comprised of the same number of dialogues, the only difference being in the use of $\beta$. Table 10 shows the average results obtained from the analysis of Dialogues $A_{5}$.

Table 10. Average evaluation results (in \%) obtained by changing the strategy to select the lexical model.

\begin{tabular}{lcccc}
\hline Dialogue corpus & WA & SU & IR & TC \\
\hline Dialogues $_{5}$ (using $\beta$ only) & 81.40 & 65.61 & 11.43 & 60.89 \\
\hline
\end{tabular}

These results are lower than those shown in Table 7 (Section 4.6.3), which indicates that using the lexical information (word confusions) associated with each prompt type $\mathrm{T}$ $\left(\mathrm{LM}_{\mathrm{T}}\right)$ for correction is better than using all the lexical information in the application domain regardless of the prompt type. This happens because the confusion probabilities of words are not the same in the $\mathrm{LM}_{\mathrm{T}}$ s $\mathrm{s}$ and in $\beta$, and these differences are in some cases deterministic in making the proper correction. For example, in accordance with the $\beta$ set used in the experiments, the highest probability of confusing the word 'error' with a word in the NUMBER concept is 0.0370 , and this word is 'dieciseis' (sixteen). However, in accordance with $\mathrm{LM}_{\mathrm{T}=\mathrm{PRODUCT}}$ ORDER, the highest probability of confusing the word 'error' with a word in the same concept is 0.0090 , and this word is 'una' (one). Therefore, if in the correction of a recognition result we assume that the word 'error' is incorrect, and we look for a word in the NUMBER concept, the selected candidate is 'dieciseis' if we consider $\beta$, and 'dos' if we take into account $\mathrm{LM}_{\mathrm{T}=\text { PRODUCT_ORDER. This shows that the }}$ lexical model employed affects the outcome of the correction process. It we compare Table 10 and Table 7 (Section 4.6.3) it follows that the best strategy to select the model for the lexical correction (either $\mathrm{LM}_{\mathrm{T}}$ or $\beta$ ) is the one proposed in this paper, which uses different models to take into account word confusion information in different contexts. 


\section{Limitations of the proposed technique}

The technique has several limitations. One is that the system designers must provide semantic information about the application domain in the form of word-classes, and linguistic information in the form of grammatical rules. This is not the case for purely statistical methods, which learn from the provided training data without requiring additional effort on the part of the system designers. Another limitation is that there are cases where the technique fails in correcting erroneous recognition results, as observed in Table 11. One failing case is when the words in the input utterance are substituted by others and the recognition result is valid in the application domain. For example, the product order "quiero una ensalada de gambas" (I want one prawn salad) was recognised as "quiero una ensalada de manzana" (I want an apple salad). In this case the ASRPC module did not made any correction because the recognition result was conceptually valid although it was incorrect. Another example is the telephone number "nueve cinco ocho setenticuatro setenticinco veintiuno" (nine five eight seventy-four seventy-five twentyone), which was recognised as "nueve cinco ocho setenticuatro sesenticinco veintiuno" (nine five eight seventy-four sixty-five twenty-one) because of the substitution of 'setenticinco' by 'sesenticinco'. Again, the ASRPC module did not make any correction because the obtained telephone number was conceptually valid although it was misrecognised.

Another failing case is when the sequence of words in the input utterance is so distorted by speech recognition errors that the obtained syntactic-semantic pattern cannot be corrected. In this case the technique either fails in the correction or does not make any correction. For example, the product order "ponme una fanta de naranja grande" (I want one large orange fanta) was recognised as "queso de bazan tercera" (cheese of 


\section{ACCEPTED MANUSCRIPT}

bazan third). For this very incorrectly recognised sentence the ASRPC did not make any

correction, as it did not find any similar pattern learnt in the training to make concept

replacements.

Table 11. Examples of unsuccessful corrections of speech recognition errors (in Spanish).

T = TELEPHONE_NUMBER

$\mathrm{U}=$ nueve cinco ocho setenticuatro setenticinco veintiuno

$h=$ nueve $(0.5999)$ cinco (1.0000) ocho (1.0000) setenticuatro (0.8000) sesenticinco $(0.4000)$ veintidos $(1.0000)$

$h^{\prime}=$ nueve $(0.5999)$ cinco $(1.0000)$ ocho $(1.0000)$ setenticuatro $(0.8000)$ sesenticinco $(0.4000)$ veintidos (1.0000)

$\mathrm{T}=$ CONFIRM_TELEPHONE_NUMBER

$\mathrm{U}=$ nueve cinco ocho setenticuatro setenticinco veintiuno

$h=$ dame $(0.6006)$ cinco $(1.0000)$ ocho (1.0000) setenticuatro $(0.5000)$ veinticinco $(0.1999)$ veintiuno (1.0000)

$h^{\prime}=$ nueve $(1.0000)$ cinco $(1.0000)$ ocho $(1.0000)$ setenticuatro $(0.5000)$ veinticinco $(0.1999)$ veintiuno (1.0000)

$\mathrm{T}=$ ADDRESS

$\mathrm{U}=$ calle acera del triunfo número dos segundo letra a

$h=\mathbf{c}(0.7983)$ diez $(0.2010)$ noveno $(0.2013)$ segundo $(0.6038)$ letra $(0.6188)$ a $(0.6038)$

$h '=\mathbf{c}(0.7983)$ diez $(0.2010)$ noveno $(0.2013)$ segundo $(0.6038)$ letra $(0.6188)$ a $(0.6038)$

T = FOOD_ORDER

$\mathrm{U}=$ ponme una fanta de naranja grande

$h=$ queso $(0.1010)$ de $(0.9005)$ bazan $(0.7013)$ tercera $(0.7013)$

$h^{\prime}=$ queso $(0.1010)$ de $(0.9005)$ bazan $(0.7013)$ tercera $(0.7013)$

$\mathrm{T}=$ TELEPHONE_NUMBER

$\mathrm{U}=$ nueve cinco ocho tres dos cero tres uno siete

$h=$ nueve $(0.6450)$ cinco $(0.7941)$ ocho (0.6019) queso (0.4002) cero (1.000) tres (0.8998) uno (1.0000) siete (1.0000)

$h^{\prime}=$ nueve $(0.6450)$ cinco $(0.7941)$ ocho $(0.6019)$ cero $(0.5392)$ tres $(0.8998)$ uno $(1.0000)$

T $=$ TELEPHONE_NUMBER

$\mathrm{U}=$ nueve cinco ocho uno tres cinco uno cinco seis

$h=$ nueve $(0.7002)$ cinco $(0.9000)$ chocolate $(0.5995)$ curry $(0.6994)$ trece $(0.5995)$

$h^{\prime}=$ nueve $(0.7002)$ cinco $(0.9000)$ trece $(0.5995)$

$\mathrm{T}=$ ARDES

$\mathrm{U}=$ calle alhóndiga número cuatro

$h=$ queremos $(0.5013)$ de $(0.8056)$ lomo $(0.4063)$ con $(0.4063)$ queso $(0.4063)$

$h '=$ queremos $(0.5013)$ de $(0.8056)$ lomo $(0.4063)$ con $(0.4063)$ queso $(0.4063)$

T = PRODUCT_ORDER

$\mathrm{U}=$ quiero una ensalada de gambas

$h=$ quiero (0.5056) una (1.0000) ensalada (0.9012) de (0.9005) manzana $(0.6924)$

$h^{\prime}=$ quiero (0.5056) una (1.0000) ensalada (0.9012) de (0.9005) manzana (0.6924)

$\mathrm{T}=$ PRODUCT_ORDER

$\mathrm{U}=$ quiero una ensalada de gambas

$h=$ de (1.0000) manzana (1.0000) manzana (0.5008)

$h^{\prime}=$ de (1.0000) manzana (1.0000) manzana (0.5008) 
Another limitation is the well-known problem of out-of-vocabulary words (OOV), which exists whenever a speech recogniser is used. All the words uttered by the speakers who recorded the speech database were included in the dictionary of the Saplen system employed in the experiments. Hence, there were no OOV problems in the testing of the technique. However, in a real interaction there may be OOV words. The effect of these words in the proposed technique depends on whether these words are keywords or not, and on the speech recognition errors that they cause.

If the OOV words are not keywords it is not important for the dialogue system to recognise the words, as they do not affect the semantics of the sentences. However, the uttering of these words may cause in-vocabulary words to be inserted, substituted or deleted in the recognition results. In terms of speech understanding, there is no problem if these in-vocabulary words are not keywords as the concepts in the utterances are not changed. The problem is when the words are keywords. The performance of the technique in dealing with in-vocabulary keyword recognition errors has been discussed in Section 3.2.3.

If the OOV words are keywords it would be important for the dialogue system to recognise the words, given that they affect the semantics of the sentences. However, these words cannot be recognised as they are unknown for the recogniser, and thus the speech recognition errors they cause cannot be corrected. This happens because the syntactic-semantic and lexical models obtained from the training cannot learn any information about these words. Hence, it is not possible to make either concept or word replacements to correct the errors. It is impossible as well to make corrections at the linguistic level given that words do not appear in the recognition results, and thus the grammatical rules are not applicable for them. 
If we wanted to check the effect of OOV words on the proposed technique employing our experimental setting, we could remove some words from the Saplen system's dictionary, which would now be OOV words. Another method would be to record new utterances which include OOV words, and create new scenarios for the user simulator that include as goals the frames associated with these new utterances. The dialogue system would analyse the new utterances when the simulator employs the new scenarios. In both cases the speech recogniser would face the problem of unknown words, which would enable recognition errors to be handled by the technique.

\section{Conclusions and future work}

This paper has presented a novel technique for ASR post-correction in spoken dialogue systems that employs semantic, syntactic, lexical and contextual information. The semantic and syntactic information is implemented by means of sets of patterns, which are created from the analysis of a dialogue corpus. Each set of patterns is mapped to a prompt type of a spoken dialogue system. The prompt type represents the contextual information of the technique, as it determines the kinds of sentence likely to be uttered by the user at a particular dialogue state. The lexical information is implemented by means of word confusion probabilities and basic grammatical rules. The confusion probabilities are computed from the analysis of the dialogue corpus, by aligning utterance transcriptions and speech recognition results. The grammatical rules are provided by the system designers and are used to ensure grammatical restrictions between specific words. These rules are necessary to compensate for problems arising from sparse training data.

The technique presents two main novel contributions to research. On the one hand, it considers several contexts where a speech recognition result can be corrected. A 
similarity threshold is used to decide whether the correction must be carried out in the context associated with the current prompt type generated by the dialogue system, or in another context. The optimal value of this threshold must be determined experimentally. On the other hand, the technique deals with the confidence scores of the words employed for the corrections. We have proposed a simple method to compute these scores that worked well in the experiments, but we do not claim that it is optimal.

Experiments to test the technique have been carried out employing a dialogue system and a user simulator, both developed in previous studies. The dialogue system used one prompt-independent language model (word bigram) for speech recognition, which enabled, in theory, the recognition of any kind of sentence permitted in the application domain, regardless of the current prompt of the dialogue system. We observed in previous experiments that this language model caused many erroneous recognition results. Hence, we used the proposed technique to try to correct some of these errors. We compared two speech recognition front-ends: i) the HTK-based speech recogniser of the dialogue system (baseline), and ii) the HTK-based recogniser and the ASRPC module, which implements the proposed technique. The results obtained, shown in Table 6 (baseline) and Table 7 (proposed technique), indicate that the technique was very useful in correcting speech recognition errors, as the system's word accuracy, speech understanding, implicit recovery and task completion rates increased by $8.5 \%, 16.54 \%, 4 \%$, and $44.17 \%$, respectively.

We also carried out experiments to check the effect on the correction process of using the contextual information provided by the system prompt in terms of syntacticsemantic models $\left(\mathrm{SSM}_{\mathrm{T}}\right.$ 's) and lexical models $\left(\mathrm{LM}_{\mathrm{T}}\right.$ 's). Regarding the former models, comparison of Table 7 and Table 9 shows that using SSM $_{\mathrm{T}}$ 's with a similarity threshold 
$t$ provides better results than: i) using the set of these models $(\alpha)$ only, and ii) using the $\mathrm{SSM}_{\mathrm{T}}$ 's if the patterns are found there, otherwise using the $\alpha$ set as a fallback strategy without considering the similarity threshold. Regarding the lexical models, comparison of Table 7 and Table 10 shows that using $\mathrm{LM}_{\mathrm{T}}$ 's if possible, and otherwise the set of these models ( $\beta$ ) as a fallback strategy, is also preferable to using $\beta$ only.

Future work includes testing the proposed technique with dialogue systems developed for other application domains, for example the Viajero system that we developed in a previous study to provide bus travel information (López-Cózar et al. 2000). Another line of research is concerned with studying other methods to decide the confidence score for the correction word. For example, one alternative strategy might be to consider the score proportional to the number of words in the concept that contains the word with which the correction word is confused. Another method would be to consider it proportional to the number of phonemes in common between the corrected word and the correction word.

\section{Acknowledgements}

The authors with to thank the reviewers for their valuable comments that have enhanced this paper.

\section{References}

Allen J. 1995. Natural Language Understanding. The Benjamin/Cummings Publishing Company Inc.

Billi, R., Castagneri, G., Danielli, M. 1997. Field trial evaluations of two different information inquiry systems. Speech Communication, vol. 23, no. 1-2, pp. 83-93

Crestani, F. 2000. Word recognition errors and relevance feedback in spoken query processing. Proc. of Conf. Flexible Query Answering Systems, pp. 267-281

Danieli, M., Gerbino, E. 1995. Metrics for evaluating dialogue strategies in a spoken language system. AAAI Spring Symposium on Empirical Methods in Discourse Interpretation and Generation, pp. 34-39 
Denda, Y., Tanaka, T., Nakayama, M., Nishiura, T., Yamashita, Y. 2007. Noise-robust hands-free voice activity detection with adaptive zero crossing detection using talker direction estimation. Proc. of ICSLP, pp. 222-225

Fisher, W. M., Fiscus, J. G. 1993. Better alignment procedures for speech recognition evaluation. Proc. of ICASSP, pp. 59-62

Hazen, T. J., Seneff, S., Polifroni, J. 2002. Recognition confidence scoring and its use in speech understanding systems. Computer Speech and Language, vol. 16, pp. 49-67

Huang, X., Acero, A., Hon, H. 2001. Spoken Language Processing: A Guide to Theory, Algorithm and System Development. Prentice-Hall, 2001

Jeong, M., Kim., B., Lee, G. G. 1996. Semantic-oriented error correction for spoken query processing. Proc. of ICSLP, pp. 897-900

Jeong, M., Jung, S., Lee, G. G. 2004. Speech recognition error correction using maximum entropy language model. Proc. of Interspeech, pp. 2137-2140

Kaki, S., Sumita, E., Iida, H. 1998. A method for correcting speech recognitions using the statistical features of character co-occurrences. Proc. of COLING-ACL, pp. 653657

Kakutani, N., Kitaoka, N., Nakagawa, S. 2002. Detection and recognition of repaired speech on misrecognized utterances for speech input of car navigation system. Proc. of ICSLP, pp.833-836

Kellner, A., Rueber, B., Seide, F., Tran, B.-H. 1997. PADIS - An automatic telephone switchboard and directory information system. Speech Communication, vol. 23, pp. 95-111

Kraiss, K. F. (Ed.). 2006. Advanced Man-Machine Interaction: Fundamentals and Implementation. Springer

Lee, C.-H., Carpenter, B., Chou, W., Chu-Carroll, J., Reichl, W., Saad, A., Zhou, Q. 2000. On natural language call routing. Speech Communication, vol. 31, pp. 309320

Levow, G. A. 1998. Characterizing and recognizing spoken corrections in humancomputer dialogue. Proc. of COLING-ACL, pp.736-742

Levow, G.-A. 2002. Adaptation in spoken corrections: Implications for models of conversational speech. Speech Communication, vol.36, pp.147-163

Lo, W. K., Soong, F. K. 2005. Generalized posterior probability for minimum error verification of recognized sentences. Proc. of ICASSP, pp. 85-88

López-Cózar, R., García, P., Díaz, J., Rubio, A. J. 1997. A voice activated dialogue system for fast-food restaurant applications. Proc. of Eurospeech, pp. 1783-1786

López-Cózar, R., Rubio, A. J., García, P., Segura, J. C. 1998. A spoken dialogue system based on a dialogue corpus analysis. Proc. of LREC, pp. 55-58

López-Cózar, R., Rubio, A. J., García, P., Díaz-Verdejo, J. E., López-Soler, J. M. 2000. Sistema de diálogo oral para proporcionar información sobre viajes en autobús (Spoken dialogue system to provide bus information). Proc. of First Spanish Workshop on Speech Technologies, Seville, Spain 
López-Cózar, R., De la Torre, A., Segura, J. C., Rubio, A. J., Sánchez, V. 2003. Assessment of dialogue systems by means of a new simulation technique. Speech Communication, 40(3), pp. 387-407

López-Cózar, R., Araki, M. 2005. Spoken, Multilingual and Multimodal Dialogue Systems. Development and Assessment. John Wiley \& Sons Publishers

López-Cózar, R., Callejas, Z. 2005. Combining Language Models in the Input Interface of a Spoken Dialogue System. Computer Speech and Language, vol. 20, pp. 420440

López-Cózar, R., Callejas, Z., McTear, M. F. 2007. Testing the performance of spoken dialogue systems by means of a new artificially-simulated user. Accepted in Artificial Intelligence Review, forthcoming

Mangu, L., Padmanabhan, M. 2001. Error corrective mechanisms for speech recognition. Proc. of ICASSP, pp. 29-32

Morales, N., Gu, L., Gao, Y. 2007. Adding noise to improve noise robustness in speech recognition. Proc. of ICSLP, pp. 930-933

McTear, M. F. Spoken dialogue technology. Toward the conversational user interface. Springer, 2004

Nakano, N., Minami, Y., Seneff, S., Hazen, T. J., Cyphers, D. S., Glass, J., Polifroni, J., Zue, V. 2001. Mokusei: A telephone-based Japanese conversational system in the weather domain. Proc. of Eurospeech, pp. 1331-1334

Ogata, J., Goto, M. 2005. Speech repair: Quick error correction just by using selection operation for speech input interfaces. Proc. of Interspeech, pp. 133-136

Rabiner, L., Juang, B.H. 1993. Fundamentals of Speech Recognition. Prentice-Hall

Ringger, E. K., Allen, J. F. 1996. A fertility model for post correction of continuous speech recognition. Proc. of ICSLP, pp. 897-900

Seneff S., Polifroni J. 2000. Dialogue management in the Mercury flight reservation system. Proc. of ANLP-NAACL Satellite Workshop, pp. 1-6

Seto, S., Kanazawa, H., Shinchi, H., Takebayashi, Y. 1994. Spontaneous speech dialogue system TOSBURG II and its evaluation. Speech Communication, vol. 15, pp. 341-353

Shi, Y., Zhou, L. 2006. Examining knowledge sources for human error correction. Proc. of Interspeech, pp. 1089-1092

Skantze, G. 2005. Exploring human error recovery strategies: Implications for Spoken Dialogue systems. Speech Communication, vol. 45, pp. 325-341

Suhm, B., Myers, B., Waibel, A. 2001. Multimodal error correction for speech user interfaces. ACM Transactions on Computer-Human Interaction, vol. 8(1), pp. 60-98

Swerts, M., Litman, D., Hirschberg, J. 2000. Correction in spoken dialogue system, Proc. of ICSLP, pp. 615-618

Wahlster, W. (Ed.) 2006. SmartKom: Foundations of Multimodal Dialogue Systems. Springer 
Ward, W., Issar, S. 1996. A class based model for speech recognition. Proc. of ICASSP, pp. 416-418

Zhou, Z., Meng, H. 2004. A two-level schemata for detecting recognition errors. Proc. of ICSLP 2004, pp. 449-452

Zhou, Z., Meng, H., Lo, W. K. 2006. A multi-pass error detection and correction framework for Mandarin LVCSR. Proc. of ICSLP, pp. 1646-1649

Zue, V., Seneff, S., Glass, J., Polifroni, J., Pao, C., Hazen, T., Hetherington, L. 2000. Jupiter: A telephone-based conversational interface for weather information. IEEE Trans. on Speech and Audio Proc., vol. 8(1), pp. 85-96 